\title{
Some Properties of the Wishart Processes and a Matrix Extension of the Hartman-Watson Laws $^{\dagger}$
}

\author{
By \\ Catherine Donati-Martin*, Yan Doumerc**, Hiroyuki Matsumoto*** \\ and Marc YOR*
}

\begin{abstract}
The aim of this paper is to discuss for Wishart processes some properties which are analogues of the corresponding well-known ones for Bessel processes. In fact, we mainly concentrate on the local absolute continuity relationship between the laws of Wishart processes with different dimensions, a property which, in the case of Bessel processes, has proven to play a rather important role in a number of applications.
\end{abstract}

\section{§1. Introduction and Main Results}

(1.0) To begin with, we introduce some notations concerning sets of matrices:

- $M_{n, m}(\mathbb{R}), M_{n, m}(\mathbb{C})$ : the set of $n \times m$ real and complex matrices

Communicated by Y. Takahashi. Received December 24, 2003.

2000 Mathematics Subject Classification(s): 60J60, 60J65, 15A52

Key words: Bessel processes, Wishart processes, Time inversion, Hartman-Watson distributions.

${ }^{\dagger}$ This article is an invited contribution to a special issue of Publications of RIMS commemorating the fortieth anniversary of the founding of the Research Institute for Mathematical Sciences.

* Laboratoire de Probabilités et Modèles aléatoires, Université P. et M. Curie, 4, place Jussieu, 75252 Paris Cedex 05, France.

e-mail: donati@ccr.jussieu.fr

** Laboratoire de Statistique et Probabilités, Université P. Sabatier, 118, route de Narbonne, 31062 Toulouse Cedex 04, France.

e-mail: doumerc@cict.fr

*** Graduate School of Information Science, Nagoya University, Chikusa-ku, Nagoya 4648601, Japan.

e-mail: matsu@info.human.nagoya-u.ac.jp

(C) 2004 Research Institute for Mathematical Sciences, Kyoto University. All rights reserved. 
- $\mathcal{S}_{m}(\mathbb{R}), \mathcal{S}_{m}(\mathbb{C})$ : the set of $m \times m$ real and complex symmetric (not selfadjoint) matrices

- $\mathcal{S}_{m}^{+}$: the set of $m \times m$ real non-negative definite matrices

- $\widetilde{\mathcal{S}}_{m}^{+}$: the set of $m \times m$ real strictly positive definite matrices

- For $A \in M_{n, m}(\mathbb{R}), A^{\prime}$ denotes its transpose. Note that $\hat{A} \stackrel{\text { def }}{=} A^{\prime} A \in \mathcal{S}_{m}^{+}$.

(1.1) The present paper constitutes a modest contribution to the studies of matrix valued diffusions which are being undertaken in recent years, due to the growing interest in random matrices; see O'Connell [22] for some recent survey. More precisely, we engage here in finding some analogues for Wishart processes of certain important properties for squared Bessel processes, which we now recall (for some similar efforts concerning the Bessel processes, see [39], pp. 64-67 and [11]).

(1.a) Definition of BESQ processes For $x \geqq 0$ and $\delta \geqq 0$, the stochastic differential equation

$$
d X_{t}=2 \sqrt{X_{t}} d B_{t}+\delta d t, \quad X_{0}=x,
$$

with the constraint $X_{t} \geqq 0$ admits one and only one solution, i.e., (1.1) enjoys pathwise uniqueness. The process is called a squared Bessel process, denoted as $\operatorname{BESQ}(\delta)$, and its distribution on the canonical space $C\left(\mathbb{R}_{+}, \mathbb{R}_{+}\right)$is denoted by $Q_{x}^{\delta}$, where, abusing the notation, we shall still denote the process of coordinates by $X_{t}, t \geqq 0$, and its filtration by $\mathcal{X}_{t}=\sigma\left\{X_{s}, s \leqq t\right\}$.

The family $\left\{Q_{x}^{\delta}\right\}_{\delta \geqq 0, x \geqq 0}$ enjoys a number of remarkable properties, among which

(1.b) Additivity property of BESQ laws We have

$$
Q_{x}^{\delta} * Q_{x^{\prime}}^{\delta^{\prime}}=Q_{x+x^{\prime}}^{\delta+\delta^{\prime}}
$$

for every $\delta, \delta^{\prime}, x, x^{\prime} \geqq 0$. This property was found by Shiga-Watanabe [31] and considered by Pitman-Yor [28] who established a Lévy-Khintchine type representation of (each of) the infinitely divisible $Q_{x}^{\delta}$ 's.

(1.c) Local absolute continuity property Writing $\delta=2(1+\nu)$, with $\nu \geqq$ -1 , and $Q_{x}^{\delta}=Q_{x}^{(\nu)}$, there is the relationship: for $\nu \geqq 0$,

$$
\left.Q_{x}^{(\nu)}\right|_{\mathcal{X}_{t}}=\left.\left(\frac{X_{t}}{x}\right)^{\nu / 2} \exp \left(-\frac{\nu^{2}}{2} \int_{0}^{t} \frac{d s}{X_{s}}\right) \cdot Q_{x}^{(0)}\right|_{\mathcal{X}_{t}},
$$

from which we can deduce that the $Q_{x}^{(0)}$-conditional law of $\int_{0}^{t}\left(X_{s}\right)^{-1} d s$ given $X_{t}=y$ is the Hartman-Watson distribution $\eta_{r}(d u), r>0, u>0$. It is 
characterized by

$$
\int_{0}^{\infty} \exp \left(-\frac{\nu^{2} u}{2}\right) \eta_{r}(d u)=\frac{I_{\nu}(r)}{I_{0}(r)}
$$

where $I_{\nu}$ denotes the usual modified Bessel function; precisely, there is the following consequence of (1.3): for $\nu \geqq 0$,

$$
Q_{x}^{(0)}\left[\exp \left(-\frac{\nu^{2}}{2} \int_{0}^{t} \frac{d s}{X_{s}}\right) \mid X_{t}=y\right]=\frac{I_{\nu}(r)}{I_{0}(r)},
$$

where $r=\sqrt{x y} / t$, and more generally,

$$
Q_{x}^{(\nu)}\left[\exp \left(-\frac{\mu^{2}}{2} \int_{0}^{t} \frac{d s}{X_{s}}\right) \mid X_{t}=y\right]=\frac{I \sqrt{\nu^{2}+\mu^{2}}(r)}{I_{\nu}(r)} .
$$

The relation (1.3) was obtained and exploited by Yor [36] to yield, in particular, the distribution at time $t$ of a continuous determination $\theta_{t}$ of the angular argument of planar Brownian motion, thus recovering previous calculations by Spitzer [32], from which one may derive Spitzer's celebrated limit law for $\theta_{t}$ :

$$
\frac{2 \theta_{t}}{\ln (t)} \stackrel{\text { (law) }}{\longrightarrow} C_{1} \quad \text { as } t \rightarrow \infty
$$

where $C_{1}$ denotes the standard Cauchy variable, with parameter 1 . It is also known that

$$
\frac{4}{(\ln (t))^{2}} \int_{0}^{t} \frac{d s}{X_{s}} \stackrel{\text { (law) }}{\longrightarrow} T_{(1 / 2)} \quad \text { as } \quad t \rightarrow \infty,
$$

where $T_{(1 / 2)}$ denotes the standard stable $(1 / 2)$ variable. We recall that

$$
E\left[\exp \left(i \lambda C_{1}\right)\right]=E\left[\exp \left(-\frac{\lambda^{2}}{2} T_{(1 / 2)}\right)\right]=\exp (-|\lambda|), \lambda \in \mathbb{R} .
$$

The absolute continuity property (1.3) has been of some use in a number of problems, see, e.g., Kendall [17] for the computation of a shape distribution for triangles, Geman-Yor [11] for the pricing of Asian options, Hirsch-Song [16] in connection with the flows of Bessel processes, and more recent work by Werner [34] who deduces the computation of Brownian intersection exponents also from the relationship (1.3).

(1.d) Time inversion Let $X_{t}$ be a $Q_{x}^{\delta}$ distributed process and define $i(X)_{t}=$ $t^{2} X(1 / t)$, then $i(X)$ is a generalized squared Bessel process with drift $\sqrt{x}$, starting from 0. (See [33] and [27] for the definitions of generalized Bessel processes). As an application, Pitman and Yor [26] give a "forward" skew product representation for the $d$-dimensional Brownian motion with drift. 
(1.e) Intertwining property If $Q_{t}^{\delta}(x, d y)$ denotes the semigroup of the $\operatorname{BESQ}(\delta)$ process, there is the intertwining relation

$$
Q_{t}^{\delta+\delta^{\prime}} \Lambda_{\delta, \delta^{\prime}}=\Lambda_{\delta, \delta^{\prime}} Q_{t}^{\delta}
$$

where $\Lambda_{\delta, \delta^{\prime}}$ denotes the multiplication operator associated with $\beta_{\delta / 2, \delta^{\prime} / 2}$, a beta variable with parameter $\left(\delta / 2, \delta^{\prime} / 2\right)$, i.e.,

$$
\Lambda_{\delta, \delta^{\prime}} f(x)=E\left[f\left(x \beta_{\delta / 2, \delta^{\prime} / 2}\right)\right]
$$

for every Borel function $f: \mathbb{R}_{+} \rightarrow \mathbb{R}_{+}$. The relation (1.7) may be proven purely in an analytical manner, but it may also be shown in a more probabilistic way, with the help of time inversion, using a realization of $X^{\delta+\delta^{\prime}}$ as the sum $X^{\delta}+X^{\delta^{\prime}}$ of two independent BESQ processes (see [7] for details).

(1.2) With the help of the above presentation of the BESQ processes, it is not difficult to discuss and summarize the main results obtained so far by M.F. Bru $([3,5])$ concerning the family of Wishart processes, which take values in $\mathcal{S}_{m}^{+}$for some $m \in \mathbb{N}$, to be fixed throughout the sequel.

For values of $\delta$ to be discussed later, $\operatorname{WIS}(\delta, m, x)$ shall denote such a Wishart process with "dimension" $\delta$, starting at $x$, to be defined as the solution of the following stochastic differential equation:

$$
d X_{t}=\sqrt{X_{t}} d B_{t}+d B_{t}^{\prime} \sqrt{X_{t}}+\delta I_{m} d t, \quad X_{0}=x,
$$

where $\left\{B_{t}, t \geqq 0\right\}$ is an $m \times m$ Brownian matrix whose components are independent one-dimensional Brownian motions, and $I_{m}$ is the identity matrix in $\mathbb{R}^{m}$. We denote the distribution of $\operatorname{WIS}(\delta, m, x)$ on $C\left(\mathbb{R}_{+}, \mathcal{S}_{m}^{+}\right)$by $\mathbf{Q}_{x}^{\delta}$.

Assume that $x \in \mathcal{S}_{m}^{+}$and that $x$ has distinct eigenvalues, which we denote by $\lambda_{1}(0)>\cdots>\lambda_{m}(0) \geqq 0$. Then, M.F. Bru [5] has shown the following

Theorem 1.1. (i) If $\delta \in(m-1, m+1)$, then (1.8) has a unique solution in $\mathcal{S}_{m}^{+}$in the sense of probability law.

(ii) If $\delta \geqq m+1$, then (1.8) has a unique strong solution in $\tilde{\mathcal{S}}_{m}^{+}$.

(iii) The eigenvalue process $\left\{\lambda_{i}(t), t \geqq 0,1 \leq i \leq m\right\}$ never collide, that is, almost surely,

$$
\lambda_{1}(t)>\cdots>\lambda_{m}(t) \geqq 0, \quad \forall t>0 .
$$

Moreover, if $\delta \geqq m+1$, then $\lambda_{m}(t)>0$ for all $t>0$ almost surely and the 
eigenvalues satisfy the stochastic differential equation

$$
\begin{aligned}
d \lambda_{i}(t) & =2 \sqrt{\lambda_{i}(t)} d \beta_{i}(t)+\left\{\delta+\sum_{k \neq i} \frac{\lambda_{i}(t)+\lambda_{k}(t)}{\lambda_{i}(t)-\lambda_{k}(t)}\right\} d t, \quad i=1, \ldots, m \\
& =2 \sqrt{\lambda_{i}(t)} d \beta_{i}(t)+\left\{\delta-m+1+2 \sum_{k \neq i} \frac{\lambda_{i}(t)}{\lambda_{i}(t)-\lambda_{k}(t)}\right\} d t
\end{aligned}
$$

where $\beta_{1}(t), \ldots, \beta_{m}(t)$ are independent Brownian motions.

(iv) If $\delta \geqq m+1$, then

$$
d\left(\operatorname{det}\left(X_{t}\right)\right)=2 \operatorname{det}\left(X_{t}\right) \sqrt{\operatorname{Tr}\left(X_{t}^{-1}\right)} d \beta(t)+(\delta-m+1) \operatorname{det}\left(X_{t}\right) \operatorname{Tr}\left(X_{t}^{-1}\right) d t
$$

and

$$
d\left(\log \left(\operatorname{det}\left(X_{t}\right)\right)\right)=2 \sqrt{\operatorname{Tr}\left(X_{t}^{-1}\right)} d \beta(t)+(\delta-m-1) \operatorname{Tr}\left(X_{t}^{-1}\right) d t,
$$

where $\beta=\{\beta(t), t \geqq 0\}$ is a Brownian motion.

(v) For any $\Theta \in \mathcal{S}_{m}^{+}$,

$$
\begin{aligned}
\mathbf{Q}_{x}^{\delta}[ & \left.\exp \left(-\operatorname{Tr}\left(\Theta X_{t}\right)\right)\right] \\
& =(\operatorname{det}(I+2 t \Theta))^{-\delta / 2} \exp \left(-\operatorname{Tr}\left(x(I+2 t \Theta)^{-1} \Theta\right)\right) \\
& =\exp (-\operatorname{Tr}(x / 2 t))(\operatorname{det}(I+2 t \Theta))^{-\delta / 2} \exp \left(\frac{1}{2 t} \operatorname{Tr}\left(x(I+2 t \Theta)^{-1}\right)\right) .
\end{aligned}
$$

For the sake of clarity, we postpone the discussion of further properties of Wishart processes as presented in M.F. Bru [5] to Section 2.

(1.3) We now present some of our main results and, in particular, the extension for Wishart processes of the absolute continuity property (1.3).

Theorem 1.2. With the above notation, we have for $\nu \geqq 0$ :

$$
\left.\mathbf{Q}_{x}^{m+1+2 \nu}\right|_{\mathcal{F}_{t}}=\left.\left(\frac{\operatorname{det}\left(X_{t}\right)}{\operatorname{det}(x)}\right)^{\nu / 2} \exp \left(-\frac{\nu^{2}}{2} \int_{0}^{t} \operatorname{Tr}\left(X_{s}^{-1}\right) d s\right) \cdot \mathbf{Q}_{x}^{m+1}\right|_{\mathcal{F}_{t}}
$$

Just as in the case of squared Bessel processes, the semigroup of $\operatorname{WIS}(\delta, m, x)$ is explicitly known, and we deduce from Theorem 1.2 our main result in this paper: 
Corollary 1.3. $\quad$ Let $\nu \geqq 0$. Then we have

$$
\begin{aligned}
\mathbf{Q}_{x}^{m+1}\left[\exp \left(-\frac{\nu^{2}}{2} \int_{0}^{t} \operatorname{Tr}\left(X_{s}^{-1}\right) d s\right) \mid X_{t}=y\right]=\left(\frac{\operatorname{det}(x)}{\operatorname{det}(y)}\right)^{\nu / 2} \frac{\mathbf{q}_{t}^{(\nu)}(x, y)}{\mathbf{q}_{t}^{(0)}(x, y)} \\
=\frac{\boldsymbol{\Gamma}_{m}((m+1) / 2)}{\boldsymbol{\Gamma}_{m}((m+1) / 2+\nu)}(\operatorname{det}(z))^{\nu / 2} \frac{{ }_{0} \mathbf{F}_{1}((m+1) / 2+\nu ; z)}{{ }_{0} \mathbf{F}_{1}((m+1) / 2 ; z)} \\
=\frac{\widetilde{\mathbf{I}}_{\nu}(z)}{\widetilde{\mathbf{I}}_{0}(z)}
\end{aligned}
$$

where $z=x y / 4 t^{2}, \mathbf{q}_{t}^{(\nu)}$ denotes the transition probability of the Wishart process of dimension $\delta=m+1+2 \nu, \boldsymbol{\Gamma}_{m}$ is the multivariate gamma function, ${ }_{0} \mathbf{F}_{1}$ is a hypergeometric function (see the appendix for the definitions of $\boldsymbol{\Gamma}_{m}$ and ${ }_{0} \mathbf{F}_{1}$ ) and $\widetilde{\mathbf{I}}_{\nu}(z)$ is the function defined by

$$
\widetilde{\mathbf{I}}_{\nu}(z)=\frac{(\operatorname{det}(z))^{\nu / 2}}{\boldsymbol{\Gamma}_{m}((m+1) / 2+\nu)}{ }_{0} \mathbf{F}_{1}((m+1) / 2+\nu ; z) .
$$

Note that in the case $m=1, \widetilde{\mathbf{I}}_{\nu}(z)$ is related to the usual modified Bessel function $I_{\nu}(z)$ (see [19]) by $\widetilde{\mathbf{I}}_{\nu}(z)=I_{\nu}\left(2 z^{1 / 2}\right)$. Clearly, formula (1.14) appears as a generalization of the result (1.4) for $m=1$.

Notation: In general, quantities related to Wishart processes will appear in boldface.

Proofs and extensions of (1.13), with two general dimensions instead of $m+1$ and $m+1+2 \nu$, are given in Section 2 .

As in the case of the Bessel processes, we obtain the absolute continuity relationship for the negative indexes in the following way.

Theorem 1.4. Assume $0<\nu<1$ and let $T_{0}$ be the first hitting time of 0 for $\left\{\operatorname{det}\left(X_{t}\right)\right\}$. Then we have

$$
\begin{aligned}
\left.\mathbf{Q}_{x}^{m+1-2 \nu}\right|_{\mathcal{F}_{t} \bigcap\left\{t<T_{0}\right\}} & =\left.\left(\frac{\operatorname{det}\left(X_{t}\right)}{\operatorname{det}(x)}\right)^{-\nu / 2} \exp \left(-\frac{\nu^{2}}{2} \int_{0}^{t} \operatorname{Tr}\left(X_{s}^{-1}\right) d s\right) \cdot \mathbf{Q}_{x}^{m+1}\right|_{\mathcal{F}_{t}} \\
& =\left.\left(\frac{\operatorname{det}\left(X_{t}\right)}{\operatorname{det}(x)}\right)^{-\nu} \cdot \mathbf{Q}_{x}^{m+1+2 \nu}\right|_{\mathcal{F}_{t}} .
\end{aligned}
$$

From formula (1.16) we may deduce the law of $T_{0}$ for $\operatorname{WIS}(m+1-2 \nu, m, x)$, which will also be given in Section 2. In particular, we obtain: 
Corollary 1.5. For $0<\nu<1$, we have

$$
\mathbf{Q}_{x}^{(-\nu)}\left(T_{0}>t \mid X_{t}=y\right)=\left(\frac{\widetilde{\mathbf{I}}_{\nu}}{\widetilde{\mathbf{I}}_{-\nu}}\right)\left(\frac{x y}{4 t^{2}}\right) .
$$

(1.4) In this paper, we also obtain some extension of the time inversion results for Bessel processes (see (1.d)). For this, we need to introduce Wishart processes with drift. For $\delta=n$ an integer, we define a Wishart process with drift $\hat{\Theta} \equiv \Theta^{\prime} \Theta$ as the process

$$
X_{t}^{\Theta}=\left(B_{t}+\Theta t\right)^{\prime}\left(B_{t}+\Theta t\right) \equiv \widehat{B_{t}+\Theta} t
$$

where $\left\{B_{s}, s \geqq 0\right\}$ is an $n \times m$ Brownian matrix starting from 0 and $\Theta=$ $\left(\Theta_{i j}\right) \in M_{n, m}(\mathbb{R})$. Its law turns out to only depend on $\hat{\Theta}=\Theta^{\prime} \Theta$. In Section 3 , we extend the definition of these processes to a non-integer dimension $\delta$ and we show that these processes are time-inversed Wishart processes.

\section{$\S 2 . \quad$ Some Properties of Wishart Processes and Proofs of Theorems}

\section{(2.1) First properties of Wishart processes}

(2.a) Wishart processes of integral dimension In the case $\delta=n$ is an integer, WIS $(n, m, x)$ is the law of the process $\left\{X_{s}=B_{s}^{\prime} B_{s} \equiv \widehat{B}_{s}, s \geqq 0\right\}$, where $\left\{B_{s}\right\}$ is an $n \times m$ Brownian matrix starting from $B_{0}$ with $\widehat{B}_{0}=B_{0}^{\prime} B_{0}=x$.

(2.b) Transition function Let $\delta>m-1$. Formula (1.12) shows that the distribution of $X_{t}$ for fixed $t$ is the non-central Wishart distribution $W_{m}\left(\delta, t I_{m}, t^{-1} x_{1}\right)$ (Muirhead's notation), see Theorem 10.3.3 in Muirhead [21]. The transition probability density $\mathbf{q}_{\delta}(t, x, d y)$ with respect to the Lebesgue measure $d y=\prod_{i \leqq j} d y_{i j}$ of the Wishart process $\left\{X_{t}\right\}$ is thus given by

$$
\begin{aligned}
& \mathbf{q}_{\delta}(t, x, y) \\
& \quad=\frac{1}{(2 t)^{\delta m / 2} \boldsymbol{\Gamma}_{m}(\delta / 2)} \exp \left(-\frac{1}{2 t} \operatorname{Tr}(x+y)\right)(\operatorname{det}(y))^{(\delta-m-1) / 2}{ }_{0} \mathbf{F}_{1}\left(\frac{\delta}{2} ; \frac{x y}{4 t^{2}}\right) \\
& \quad=\frac{1}{(2 t)^{m(m+1) / 2}} \exp \left(-\frac{1}{2 t} \operatorname{Tr}(x+y)\right)\left(\frac{\operatorname{det}(y)}{\operatorname{det}(x)}\right)^{(\delta-m-1) / 4} \widetilde{\mathbf{I}}_{(\delta-m-1) / 2}\left(\frac{x y}{4 t^{2}}\right),
\end{aligned}
$$

where $\boldsymbol{\Gamma}_{m}$ is the multivariate gamma function, ${ }_{0} \mathbf{F}_{1}$ is a hypergeometric function (see their definitions in the appendix) and $\widetilde{\mathbf{I}}_{\nu}(z)$ is the function defined by (1.15). The transition probability density $\mathbf{q}_{\delta}(t, x, y)$ may be continuously extended in $x$ belonging to $\mathcal{S}_{m}^{+}$, and we can consider the Wishart processes starting 
from degenerate matrices. Indeed, the Wishart processes starting from 0 will play some role in the following. Note that

$$
\mathbf{q}_{\delta}(t, 0, y)=\frac{1}{(2 t)^{\delta m / 2} \boldsymbol{\Gamma}_{m}(\delta / 2)} \exp \left(-\frac{1}{2 t} \operatorname{Tr}(y)\right)(\operatorname{det}(y))^{(\delta-m-1) / 2} .
$$

(2.c) Additivity property We have the following property (see [5]): If $\left\{X_{t}\right\}$ and $\left\{Y_{t}\right\}$ are two independent Wishart processes $\operatorname{WIS}(\delta, m, x)$ and $\mathrm{WIS}\left(\delta^{\prime}, m, y\right)$, then $\left\{X_{t}+Y_{t}\right\}$ is a Wishart process $\mathrm{WIS}\left(\delta+\delta^{\prime}, m, x+y\right)$. Nevertheless, the laws $\mathbf{Q}_{x}^{\delta}$ of $\operatorname{WIS}(\delta, m, x)$ are not infinitely divisible since the parameter $\delta$ cannot take all the positive values, in fact, $\delta$ needs to belong to the so-called Gindikin's ensemble $\Lambda_{m}=\{1,2, \ldots, m-1\} \bigcup(m-1, \infty)$ (see Lévy [20] for the Wishart distribution).

(2.d) The eigenvalue process The drift in the stochastic differential equation (1.9) giving the eigenvalues of the Wishart process is a repelling force between these eigenvalues (which may be thought as positions of particles) which prohibits collisions. We now discuss some other models of non colliding processes. In [18], König and O'Connell consider the eigenvalues of the Laguerre process (defined as in (2.a) replacing the Brownian motion $B$ by a complex Brownian motion and the transpose by the adjoint for $n \geqq m$ ). Then, the eigenvalue process satisfies the same equation as (1.9) except that the drift is multiplied by " 2 ". It is shown that this process evolves like $m$ independent squared Bessel processes conditioned never to collide.

Gillet [12] considers a stochastic differential equation for an $m$-dimensional process, called a watermelon, whose paths don't intersect. It turns out that this process corresponds to the square roots of the eigenvalues of a Laguerre process and then can be interpreted as the process obtained from $m$ independent three dimensional Bessel processes conditioned to stay in the Weyl chamber $W=\left\{\left(x_{1}, x_{2}, \ldots, x_{m}\right) ; x_{1}>x_{2}>\cdots>x_{m}\right\}$

We also refer to Cépa-Lépingle [9] and Grabiner [14] for other closely related studies about non-colliding particles.

We now study the filtration of the processes which appear in the density (1.13).

Proposition 2.1. $\quad$ (i) Let $\left\{\mathcal{D}_{t}, t \geqq 0\right\}$ be the filtration generated by the process $\left\{D_{t}=\operatorname{det}\left(X_{t}\right)\right\}$. Then $\left\{\mathcal{D}_{t}\right\}$ is equal to the filtration generated by the eigenvalues $\left\{\lambda_{i}(t), i=1, \ldots, m, t \geqq 0\right\}$ of the process $\left\{X_{t}\right\}$. Therefore, the density in (1.13) is $\mathcal{D}_{t}$ measurable.

(ii) Let $\Lambda_{\bar{\lambda}}^{\delta}$ the probability law of the eigenvalues $\left(\lambda_{i}(t) ; i=1, \ldots, m\right)$ of a $\operatorname{WIS}(\delta, m, x)$ with $\bar{\lambda}$ the vector of the eigenvalues of $x$; i.e., the solution of 
(1.9) starting from $\bar{\lambda}$. Then, the absolute continuity relation (1.13) reads

$$
\left.\Lambda_{\bar{\lambda}}^{m+1+2 \nu}\right|_{\mathcal{D}_{t}}=\left.\left(\frac{\prod_{i=1}^{m} \lambda_{i}(t)}{\prod_{i=1}^{m} \lambda_{i}(0)}\right)^{\nu / 2} \exp \left(-\frac{\nu^{2}}{2} \int_{0}^{t}\left(\sum_{i=1}^{m} \frac{1}{\lambda_{i}(s)}\right) d s\right) \cdot \Lambda_{\bar{\lambda}}^{m+1}\right|_{\mathcal{D}_{t}} .
$$

Proof. (i) Denote by $L_{t}=\ln \left(D_{t}\right)=\sum_{i=1}^{m} \ln \left(\lambda_{i}(t)\right) . L_{t}$ is $\mathcal{D}_{t}$ measurable. According to equation (1.9), we have

$$
\ln \left(\lambda_{i}(t)\right)=\frac{2}{\sqrt{\lambda_{i}(t)}} d \beta_{i}(t)+K_{i}(\lambda(t)) d t
$$

for a function $K_{i}$ on $\mathbb{R}^{m}$ and

$$
\langle L, L\rangle_{t}=4 \int_{0}^{t} \sum_{i=1}^{m}\left(\frac{1}{\lambda_{i}(s)}\right) d s=4 \int_{0}^{t} \operatorname{Tr}\left(X_{s}^{-1}\right) d s,
$$

which shows that $\operatorname{Tr}\left(X_{t}^{-1}\right)=d\langle L, L\rangle_{t} / d t$ is $\mathcal{D}_{t}$ measurable.

Now, let us define $L_{p}(t)=\operatorname{Tr}\left(X_{t}^{-p}\right), p \in \mathbb{N}$ with $L_{0}(t) \equiv L(t)$. It is easy to verify that

$$
\frac{d}{d t}\left\langle L_{p}, L_{q}\right\rangle_{t}=L_{p+q+1}(t)
$$

and therefore, it follows that all the processes $L_{p}(t)=\sum_{i=0}^{n}\left(\lambda_{i}(t)\right)^{-p}$ are $\mathcal{D}_{t}$ measurable. Now, from the knowledge of all the processes $L_{p}, p \in \mathbb{N}$, we can recover the $m$-dimensional process $\left\{\lambda_{i}(t), i=1, \ldots, m, t \geqq 0\right\}$.

(ii) We just write the density in terms of the eigenvalues.

(2.2) Girsanov formula Here, after writing the Girsanov formula in our context, we prove Theorem 1.2, i.e., the absolute continuity relationship between the laws of Wishart processes of different dimensions. We also show that we may obtain, by using the Girsanov formula, a process which may be called a squared Ornstein-Uhlenbeck type Wishart process.

Let $\mathbf{Q}_{x}^{\delta}, x \in \widetilde{\mathcal{S}}_{m}^{+}, \delta>m-1$, be the probability law of $\operatorname{WIS}(\delta, m, x)$ process $\left\{X_{t}, t \geqq 0\right\}$, which is considered as the unique solution of

$$
d X_{t}=\sqrt{X_{t}} d B_{t}+d B_{t}^{\prime} \sqrt{X_{t}}+\delta I_{m} d t, \quad X_{0}=x,
$$

where $\left\{B_{t}\right\}$ is an $m \times m$ Brownian matrix under $\mathbf{Q}_{x}^{\delta}$. We consider a predictable process $H=\left\{H_{s}\right\}$, valued in $\mathcal{S}_{m}$, such that

$$
\mathcal{E}_{t}^{H}=\exp \left(\int_{0}^{t} \operatorname{Tr}\left(H_{s} d B_{s}\right)-\frac{1}{2} \int_{0}^{t} \operatorname{Tr}\left(H_{s}^{2}\right) d s\right)
$$


is a martingale with respect to $\mathbf{Q}_{x}^{\delta}$ and denote by $\mathbf{Q}_{x}^{\delta, H}$ the probability measure such that

$$
\left.\mathbf{Q}_{x}^{\delta, H}\right|_{\mathcal{F}_{t}}=\left.\mathcal{E}_{t}^{H} \cdot \mathbf{Q}_{x}^{\delta}\right|_{\mathcal{F}_{t}}
$$

where $\left\{\mathcal{F}_{t}\right\}$ is the natural filtration of $\left\{X_{t}\right\}$. Then the process $\left\{\beta_{t}\right\}$ given by

$$
\beta_{t}=B_{t}-\int_{0}^{t} H_{s} d s
$$

is a Brownian matrix under $\mathbf{Q}_{x}^{\delta, H}$ and $\left\{X_{t}\right\}$ is a solution of

$$
d X_{t}=\sqrt{X_{t}} d \beta_{t}+d \beta_{t}^{\prime} \sqrt{X_{t}}+\left(\sqrt{X_{t}} H_{t}+H_{t} \sqrt{X_{t}}+\delta I_{m}\right) d t
$$

We consider two special cases: $H_{t}=\nu X_{t}^{-1 / 2}, \nu>0$, and $H_{t}=\lambda \sqrt{X_{t}}, \lambda \in \mathbb{R}$.

Remark 2.2. Here is a slight generalization of (2.3): let $\left\{H_{s}\right\}$ be a predictable process with values in $M_{n, m}(\mathbb{R})$ and $\left\{B_{s}\right\}$ be an $n \times m$ Brownian matrix under $\mathbf{P}$. Then, under $\mathbf{P}^{H}$ given by

$$
\left.\mathbf{P}^{H}\right|_{\mathcal{F}_{t}}=\left.\exp \left(\int_{0}^{t} \operatorname{Tr}\left(H_{s}^{\prime} d B_{s}\right)-\frac{1}{2} \int_{0}^{t} \operatorname{Tr}\left(\hat{H}_{s}\right) d s\right) \cdot \mathbf{P}\right|_{\mathcal{F}_{t}},
$$

$\beta_{t}=B_{t}-\int_{0}^{t} H_{s} d s$ is an $n \times m$ Brownian matrix under $\mathbf{P}^{H}$.

Case 1 Let $H_{t}=\nu X_{t}^{-1 / 2}$. Then the equation (2.4) becomes

$$
d X_{t}=\sqrt{X_{t}} d \beta_{t}+d \beta_{t}^{\prime} \sqrt{X_{t}}+(\delta+2 \nu) I_{m} d t
$$

which is the stochastic differential equation for a $\operatorname{WIS}(\delta+2 \nu, m, x)$ process. That is, we have obtained

$$
\left.\mathbf{Q}_{x}^{\delta+2 \nu}\right|_{\mathcal{F}_{t}}=\left.\exp \left(\nu \int_{0}^{t} \operatorname{Tr}\left(X_{s}^{-1 / 2} d B_{s}\right)-\frac{\nu^{2}}{2} \int_{0}^{t} \operatorname{Tr}\left(X_{s}^{-1}\right) d s\right) \cdot \mathbf{Q}_{x}^{\delta}\right|_{\mathcal{F}_{t}} .
$$

We can write the stochastic integral on the right hand side in a simpler way when $\delta=m+1$ and thus obtain Theorem 1.2, as we now show.

Proof of Theorem 1.2. Developing the determinant of $y \in \widetilde{\mathcal{S}}_{m}^{+}$in terms of its cofactors, we obtain $\nabla_{y}(\operatorname{det}(y))=\operatorname{det}(y) y^{-1}$ and, hence,

$$
\nabla_{y}(\log (\operatorname{det}(y)))=y^{-1} .
$$


We know, from (1.11), that $\left\{\log \left(\operatorname{det}\left(X_{t}\right)\right)\right\}$ is a local martingale when $\delta=m+1$. Moreover, by (2.6), we obtain from Itô's formula

$$
\begin{aligned}
\log \left(\operatorname{det}\left(X_{t}\right)\right) & =\log (\operatorname{det}(x))+\int_{0}^{t} \operatorname{Tr}\left(X_{s}^{-1}\left(\sqrt{X_{s}} d B_{s}+d B_{s}^{\prime} \sqrt{X_{s}}\right)^{\prime}\right) \\
& =\log (\operatorname{det}(x))+2 \int_{0}^{t} \operatorname{Tr}\left(X_{s}^{-1 / 2} d B_{s}\right) .
\end{aligned}
$$

Hence, by (2.5), we obtain

$$
\left.\mathbf{Q}_{x}^{m+1+2 \nu}\right|_{\mathcal{F}_{t}}=\left.\left(\frac{\operatorname{det}\left(X_{t}\right)}{\operatorname{det}(x)}\right)^{\nu / 2} \exp \left(-\frac{\nu^{2}}{2} \int_{0}^{t} \operatorname{Tr}\left(X_{s}^{-1}\right) d s\right) \cdot \mathbf{Q}_{x}^{m+1}\right|_{\mathcal{F}_{t}} .
$$

Remark 2.3. According to Theorem 1.2, we have the following absolute continuity relationship, for $\delta=m+1+2 \lambda$ and $\delta^{\prime}=m+1+2 \nu, \lambda, \nu \geqq 0$,

$$
\mathbf{Q}_{x}^{\delta^{\prime}}{\mid \mathcal{F}_{t}}=\left.\left(\frac{\operatorname{det}\left(X_{t}\right)}{\operatorname{det}(x)}\right)^{(\nu-\lambda) / 2} \exp \left(-\frac{\nu^{2}-\lambda^{2}}{2} \int_{0}^{t} \operatorname{Tr}\left(X_{s}^{-1}\right) d s\right) \cdot \mathbf{Q}_{x}^{\delta}\right|_{\mathcal{F}_{t}}
$$

from which we deduce for $\alpha \in \mathbb{R}$

$$
\begin{aligned}
\mathbf{Q}_{x}^{\delta} & {\left[\left(\frac{\operatorname{det}\left(X_{t}\right)}{\operatorname{det}(x)}\right)^{\alpha} \exp \left(-\frac{\nu^{2}-\lambda^{2}}{2} \int_{0}^{t} \operatorname{Tr}\left(X_{s}^{-1}\right) d s\right)\right] } \\
& =\mathbf{Q}_{x}^{\delta^{\prime}}\left[\left(\frac{\operatorname{det}\left(X_{t}\right)}{\operatorname{det}(x)}\right)^{\alpha-(\nu-\lambda) / 2}\right] .
\end{aligned}
$$

The moments of $\operatorname{det}\left(X_{t}\right)$ are given by the following formula (see [21] p. 447):

$$
\mathbf{Q}_{x}^{\delta}\left[\left(\operatorname{det}\left(X_{t}\right)\right)^{s}\right]=(2 t)^{m s} \frac{\boldsymbol{\Gamma}_{m}(s+\delta / 2)}{\boldsymbol{\Gamma}_{m}(\delta / 2)}{ }_{1} \mathbf{F}_{1}\left(-s ; \frac{\delta}{2} ;-\frac{x}{2 t}\right) .
$$

For $x=0$, we have

$$
\mathbf{Q}_{0}^{\delta}\left[\left(\operatorname{det}\left(X_{t}\right)\right)^{s}\right]=(2 t)^{m s} \frac{\boldsymbol{\Gamma}_{m}(s+\delta / 2)}{\boldsymbol{\Gamma}_{m}(\delta / 2)}=(2 t)^{m s} \frac{\prod_{i=1}^{m} \Gamma(s+\delta / 2-(i-1) / 2)}{\prod_{i=1}^{m} \Gamma(\delta / 2-(i-1) / 2)}
$$

for $s>0$, which is the Mellin transform of the distribution of $\operatorname{det}\left(X_{t}\right)$ under $\mathbf{Q}_{0}^{\delta}$. Hence, letting $Y_{1}, \ldots, Y_{m}$ be independent gamma variables whose densities are given by

$$
\frac{1}{\Gamma(\delta / 2-(i-1) / 2)} e^{-\xi} \xi^{\delta / 2-(i-1) / 2-1}, \xi>0, i=1, \ldots, m,
$$

we see that the distribution of $\operatorname{det}\left(X_{t}\right)$ under $\mathbf{Q}_{0}^{\delta}$ coincides with that of $(2 t)^{m} Y_{1} \cdots Y_{m}$. This result is a consequence of Bartlett's decomposition (cf. [21, Theorem 3.2.14]). 
Case 2 Let $H_{t}=\lambda \sqrt{X_{t}}, \lambda \in \mathbb{R}$. Then (2.4) becomes

$$
d X_{t}=\sqrt{X_{t}} d \beta_{t}+d \beta_{t}^{\prime} \sqrt{X_{t}}+\left(2 \lambda X_{t}+\delta I_{m}\right) d t .
$$

By (2.2), we obtain

$$
d\left(\operatorname{Tr}\left(X_{t}\right)\right)=2 \operatorname{Tr}\left(\sqrt{X_{t}} d B_{t}\right)+m \delta d t
$$

and

$$
\int_{0}^{t} \operatorname{Tr}\left(\sqrt{X_{s}} d B_{s}\right)=\frac{1}{2}\left(\operatorname{Tr}\left(X_{t}\right)-\operatorname{Tr}(x)-m \delta t\right) .
$$

Hence, from (2.3), we have obtained that the probability measure ${ }^{\lambda} \mathbf{Q}_{x}^{\delta}$ given by

$$
\left.{ }^{\lambda} \mathbf{Q}_{x}^{\delta}\right|_{\mathcal{F}_{t}}=\exp \left(\frac{\lambda}{2}\left(\operatorname{Tr}\left(X_{t}\right)-\operatorname{Tr}(x)-m \delta t\right)-\frac{\lambda^{2}}{2} \int_{0}^{t} \operatorname{Tr}\left(X_{s}\right) d s\right) \cdot \mathbf{Q}_{x}^{\delta} \mid \mathcal{F}_{t}
$$

is the probability law of the process given by

$$
d X_{t}=\left(\sqrt{X_{t}} d \beta_{t}+d \beta_{t}^{\prime} \sqrt{X_{t}}\right)+\left(2 \lambda X_{t}+\delta I_{m}\right) d t, \quad X_{0}=x,
$$

for a Brownian matrix $\left\{\beta_{t}\right\}$ (under ${ }^{\lambda} \mathbf{Q}_{x}^{\delta}$ ). See M.F. Bru [5] for a study of squared Ornstein Uhlenbeck processes and related computations of Laplace transforms.

(2.3) Generalized Hartman-Watson laws We concentrate on the case $\delta \geqq m+1$ for a while and write $\delta=m+1+2 \nu$. We denote by $\mathbf{q}_{t}^{(\nu)}(x, y)$ the transition probability density with respect to the Lebesgue measure of the generalized Wishart process (a solution to (1.8)) $\left\{X_{t}^{(\nu)}\right\}$ given by (2.1). Then, we have

$$
\begin{aligned}
\frac{\mathbf{q}_{t}^{(\nu)}(x, y)}{\mathbf{q}_{t}^{(0)}(x, y)}= & \frac{(2 t)^{m(m+1) / 2} \boldsymbol{\Gamma}_{m}((m+1) / 2)}{(2 t)^{m(m+1+2 \nu) / 2} \boldsymbol{\Gamma}_{m}((m+1) / 2+\nu)}(\operatorname{det}(y))^{\nu} \\
& \times \frac{{ }_{0} \mathbf{F}_{1}\left((m+1) / 2+\nu ; x y / 4 t^{2}\right)}{{ }_{0} \mathbf{F}_{1}\left((m+1) / 2 ; x y / 4 t^{2}\right)} \\
= & \frac{\boldsymbol{\Gamma}_{m}((m+1) / 2)}{\boldsymbol{\Gamma}_{m}((m+1) / 2+\nu)}\left(\operatorname{det} \frac{y}{2 t}\right)^{\nu} \frac{{ }_{0} \mathbf{F}_{1}\left((m+1) / 2+\nu ; x y / 4 t^{2}\right)}{{ }_{0} \mathbf{F}_{1}\left((m+1) / 2 ; x y / 4 t^{2}\right)} .
\end{aligned}
$$

Denoting the law of $\left\{X_{t}^{(\nu)}\right\}$ by $\mathbf{Q}_{x}^{(\nu)}$, we showed in the previous subsection

$$
\left.\frac{d \mathbf{Q}_{x}^{(\nu)}}{d \mathbf{Q}_{x}^{(0)}}\right|_{\mathcal{F}_{t}}=\left(\frac{\operatorname{det}\left(X_{t}\right)}{\operatorname{det}(x)}\right)^{\nu / 2} \exp \left(-\frac{\nu^{2}}{2} \int_{0}^{t} \operatorname{Tr}\left(X_{u}^{-1}\right) d u\right),
$$


which yields

$$
\frac{\mathbf{q}_{t}^{(\nu)}(x, y)}{\mathbf{q}_{t}^{(0)}(x, y)}=\left(\frac{\operatorname{det}(y)}{\operatorname{det}(x)}\right)^{\nu / 2} \mathbf{Q}_{x}^{(0)}\left[\exp \left(-\frac{\nu^{2}}{2} \int_{0}^{t} \operatorname{Tr}\left(X_{u}^{-1}\right) d u\right) \mid X_{t}=y\right] .
$$

Therefore we obtain

$$
\begin{aligned}
\mathbf{Q}_{x}^{(0)}\left[\exp \left(-\frac{\nu^{2}}{2} \int_{0}^{t} \operatorname{Tr}\left(X_{u}^{-1}\right) d u\right) \mid X_{t}=y\right] \\
=\frac{\boldsymbol{\Gamma}_{m}((m+1) / 2)}{\boldsymbol{\Gamma}_{m}((m+1) / 2+\nu)}(\operatorname{det}(z))^{\nu / 2} \frac{{ }_{0} \mathbf{F}_{1}((m+1) / 2+\nu ; z)}{{ }_{0} \mathbf{F}_{1}((m+1) / 2 ; z)}
\end{aligned}
$$

with $z=x y / 4 t^{2}$, proving Corollary 1.3.

Using the function $\widetilde{\mathbf{I}}_{\nu}$ defined by (1.15), we may also write

$$
\mathbf{Q}_{x}^{(0)}\left[\exp \left(-\frac{\nu^{2}}{2} \int_{0}^{t} \operatorname{Tr}\left(X_{u}^{-1}\right) d u\right) \mid X_{t}=y\right]=\frac{\widetilde{\mathbf{I}}_{\nu}(z)}{\widetilde{\mathbf{I}}_{0}(z)},
$$

which is precisely (1.4) when $m=1$.

We can extend (2.12) as follows:

Proposition 2.4. Let $\lambda \geqq 0, \nu \geqq 0$,

$$
\begin{aligned}
\mathbf{Q}_{x}^{(0)} & {\left[\exp \left(-\frac{\lambda^{2}}{2} \int_{0}^{t} \operatorname{Tr}\left(X_{u}\right) d u-\frac{\nu^{2}}{2} \int_{0}^{t} \operatorname{Tr}\left(X_{u}^{-1}\right) d u\right) \mid X_{t}=y\right] } \\
& =\left(\frac{\lambda t}{\sinh (\lambda t)}\right)^{m(m+1) / 2} \exp \left(-a_{\lambda}(t) \operatorname{Tr}(x+y)\right) \frac{\widetilde{\mathbf{I}}_{\nu}\left(\lambda^{2} x y / 4 \sinh ^{2}(\lambda t)\right)}{\widetilde{\mathbf{I}}_{0}\left(x y / 4 t^{2}\right)},
\end{aligned}
$$

where $a_{\lambda}(t)=(2 t)^{-1}(\lambda t \operatorname{coth}(\lambda t)-1)$.

Remark 2.5. (i) The computation in the case $\nu=0$ was done by M.F. Bru in [5].

(ii) In the case $m=1$, formula (2.13) was obtained in [28] and yields to the joint characteristic function of the stochastic area and winding number of planar Brownian motion $\left\{Z_{u}, u \leqq t\right\}$.

Proof. From the absolute continuity relationships (1.13) and (2.8), we obtain

$$
\begin{aligned}
\left.\frac{d^{\lambda} \mathbf{Q}_{x}^{(\nu)}}{d \mathbf{Q}_{x}^{(0)}}\right|_{\mathcal{F}_{t}}= & \left(\frac{\operatorname{det}\left(X_{t}\right)}{\operatorname{det}(x)}\right)^{\nu / 2} \exp \left(\frac{\lambda}{2}\left(\operatorname{Tr}\left(X_{t}\right)-\operatorname{Tr}(x)-m \delta t\right)\right) \\
& \times \exp \left(-\frac{\lambda^{2}}{2} \int_{0}^{t} \operatorname{Tr}\left(X_{u}\right) d u-\frac{\nu^{2}}{2} \int_{0}^{t} \operatorname{Tr}\left(X_{u}^{-1}\right) d u\right),
\end{aligned}
$$


from which we deduce

$$
\begin{aligned}
\mathbf{Q}_{x}^{(0)}[\exp & \left.\left(-\frac{\lambda^{2}}{2} \int_{0}^{t} \operatorname{Tr}\left(X_{u}\right) d u-\frac{\nu^{2}}{2} \int_{0}^{t} \operatorname{Tr}\left(X_{u}^{-1}\right) d u\right) \mid X_{t}=y\right] \\
& =\frac{{ }^{\lambda} \mathbf{q}_{t}^{(\nu)}(x, y)}{\mathbf{q}_{t}^{(0)}(x, y)}\left(\frac{\operatorname{det}(x)}{\operatorname{det}(y)}\right)^{\nu / 2} \exp \left(-\frac{\lambda}{2}(\operatorname{Tr}(y)-\operatorname{Tr}(x)-m \delta t)\right),
\end{aligned}
$$

where ${ }^{\lambda} \mathbf{q}^{(\nu)}$ is the transition density of the squared Ornstein Uhlenbeck process ${ }^{\lambda} X$, the solution of $(2.9)$. Since ${ }^{\lambda} X_{t}=e^{2 \lambda t} X\left(\left(1-e^{-2 \lambda t}\right) / 2 \lambda\right)$ for some Wishart process $X$, we have

$$
{ }^{\lambda} \mathbf{q}^{(\nu)}(t, x, y)=e^{-\lambda m(m+1) t} \mathbf{q}^{(\nu)}\left(\frac{1-e^{-2 \lambda t}}{2 \lambda}, x, y e^{-2 \lambda t}\right) .
$$

Straightforward computations give (2.13).

(2.4) The case of negative indexes We first give a proof of Theorem 1.4 and then discuss the law of $T_{0}$, the first hitting time of 0 by $\left\{\operatorname{det}\left(X_{t}\right)\right\}$.

Proof of Theorem 1.4. We consider the local martingale $\left\{M_{t}\right\}$ under $\mathbf{Q}_{x}^{(0)}$ defined by

$$
M_{t}=\left(\frac{\operatorname{det}\left(X_{t}\right)}{\operatorname{det}(x)}\right)^{-\nu / 2} \exp \left(-\frac{\nu^{2}}{2} \int_{0}^{t} \operatorname{Tr}\left(X_{s}^{-1}\right) d s\right) .
$$

Note that, for $\varepsilon>0,\left\{M_{t \wedge T_{\varepsilon}}\right\}$ is a bounded martingale, where $T_{\varepsilon}=\inf$ $\left\{t ; \operatorname{det}\left(X_{t}\right) \leqq \varepsilon\right\}$. Then, applying the Girsanov theorem, we find

$$
\left.\mathbf{Q}_{x}^{(-\nu)}\right|_{\mathcal{F}_{t \wedge T_{\varepsilon}}}=\left.M_{t \wedge T_{\varepsilon}} \cdot \mathbf{Q}_{x}^{(0)}\right|_{\mathcal{F}_{t \wedge T_{\varepsilon}}}
$$

Hence, letting $\varepsilon$ tend to 0 , we obtain the result, since $T_{0}=\infty$ a.s. on the right hand side.

Proof of Corollary 1.5. From the second equality in (1.16), we obtain

$$
\mathbf{Q}_{x}^{(-\nu)}\left(T_{0}>t \mid X_{t}=y\right)=\left(\frac{\operatorname{det}(x)}{\operatorname{det}(y)}\right)^{\nu} \frac{\mathbf{q}_{t}^{(\nu)}(x, y)}{\mathbf{q}_{t}^{(-\nu)}(x, y)} .
$$

Now, using the expression of the semigroup $\mathbf{q}_{t}^{(\nu)}(x, y)$ given in (2.1), we obtain (1.17).

We next give the tail of the law of $T_{0}$ under $\mathbf{Q}_{x}^{(-\nu)}$. 
Proposition 2.6. For any $t>0$, we have

$$
\begin{aligned}
\mathbf{Q}_{x}^{(-\nu)}\left(T_{0}>t\right) & =\frac{\boldsymbol{\Gamma}_{m}((m+1) / 2)}{\boldsymbol{\Gamma}_{m}(\delta / 2)}\left(\operatorname{det}\left(\frac{x}{2 t}\right)\right)^{\nu} e^{-\operatorname{Tr}(x / 2 t)}{ }_{1} \mathbf{F}_{1}\left(\frac{m+1}{2} ; \frac{\delta}{2} ; \frac{x}{2 t}\right) \\
(2.15) \quad & =\frac{\boldsymbol{\Gamma}_{m}((m+1) / 2)}{\boldsymbol{\Gamma}_{m}(\delta / 2)}\left(\operatorname{det}\left(\frac{x}{2 t}\right)\right)^{\nu}{ }_{1} \mathbf{F}_{1}\left(\nu ; \frac{\delta}{2} ;-\frac{x}{2 t}\right),
\end{aligned}
$$

where $\delta=m+1+2 \nu$.

Proof. $\quad$ By Theorem 1.4, we have

$$
\mathbf{Q}_{x}^{(-\nu)}\left(T_{0}>t\right)=\mathbf{Q}_{x}^{(\nu)}\left[\left(\frac{\operatorname{det}(x)}{\operatorname{det}\left(X_{t}\right)}\right)^{\nu}\right]
$$

and compute the right hand side by using the explicit expression (2.1) for the semigroup of $\left\{X_{t}\right\}$.

We have by $(2.1)$

$$
\begin{aligned}
& \mathbf{Q}_{x}^{(\nu)}\left[\left(\frac{\operatorname{det}(x)}{\operatorname{det}\left(X_{t}\right)}\right)^{\nu}\right] \\
& =\frac{\exp (-\operatorname{Tr}(x) / 2 t)(\operatorname{det}(x))^{\nu}}{(2 t)^{m \delta / 2} \boldsymbol{\Gamma}_{m}(\delta / 2)} \int_{\mathcal{S}_{m}^{+}} e^{-\operatorname{Tr}(y) / 2 t}{ }_{0} \mathbf{F}_{1}\left(\frac{\delta}{2} ; \frac{x y}{4 t^{2}}\right) d y .
\end{aligned}
$$

Noting that ${ }_{0} \mathbf{F}_{1}\left(\delta / 2 ; x y / 4 t^{2}\right)={ }_{0} \mathbf{F}_{1}\left(\delta / 2 ; \sqrt{x} y \sqrt{x} / 4 t^{2}\right)$ from definition, we change the variables by $z=\sqrt{x} y \sqrt{x} / 4 t^{2}$ to obtain

$$
\begin{aligned}
\mathbf{Q}_{x}^{(\nu)} & {\left[\left(\frac{\operatorname{det}(x)}{\operatorname{det}\left(X_{t}\right)}\right)^{\nu}\right] } \\
& =\frac{\exp (-\operatorname{Tr}(x) / 2 t)(\operatorname{det}(x))^{\nu-(m+1) / 2}}{(2 t)^{m(\delta / 2-m-1)} \boldsymbol{\Gamma}_{m}(\delta / 2)} \int_{\mathcal{S}_{m}^{+}} e^{-2 t \operatorname{Tr}\left(x^{-1} z\right)}{ }_{0} \mathbf{F}_{1}\left(\frac{\delta}{2} ; z\right) d z .
\end{aligned}
$$

For the formula for the Jacobian, see Theorem 2.1.6, p.58, in [21].

Then, using the fact that the Laplace transform of a ${ }_{p} \mathbf{F}_{q}$ function is a ${ }_{p+1} \mathbf{F}_{q}$ function (cf. Theorem 7.3.4, p.260, in [21]), we get (2.14) and then, using the Kummer relation (Theorem 7.4.3, p.265, in [21]), (2.15).

Remark 2.7. When $m=1$, we can explicitly compute the right hand side of (2.16) and show that $T_{0}$ is distributed as $x / 2 \gamma_{\nu}$, where $\gamma_{\nu}$ is a gamma variable with parameter $\nu$. It may be also obtained by using the integral relation

$$
\frac{1}{X_{t}^{\nu}}=\frac{1}{\Gamma(\nu)} \int_{0}^{\infty} u^{\nu-1} e^{-u X_{t}} d u,
$$


and then the explicit expression for $Q_{x}^{(\nu)}\left[e^{-u X_{t}}\right]$. A third method consists in using the time reversal between $\operatorname{BES}(\nu)$ and $\operatorname{BES}(-\nu)$; see paper \#1 in [39] for details.

Remark 2.8. As the knowledge of the law of $T_{0}$ under $Q_{x}^{(-\nu)}$ has played an important role in several questions for $m=1$ (in the pricing of Asian options in particular, see, e.g [11]), it seems worth looking for some better expression than (2.14) or (2.15). First, let us define $S_{0}=\left(2 T_{0}\right)^{-1}$, and note that, from (2.15), we have

$$
\mathbf{Q}_{x}^{(-\nu)}\left(S_{0} \leqq u\right)=\frac{\boldsymbol{\Gamma}_{m}((m+1) / 2)}{\boldsymbol{\Gamma}_{m}(\delta / 2)}(\operatorname{det}(x))^{\nu} u^{m \nu}{ }_{1} \mathbf{F}_{1}\left(\nu ; \frac{\delta}{2} ;-u x\right) .
$$

Note in particular that the right hand side of (2.18) is a distribution function in $u$.

From (2.17), we also have the following expression

$$
\begin{aligned}
\mathbf{Q}_{x}^{(-\nu)}\left(S_{0} \leqq u\right)=\frac{(\operatorname{det}(x))^{\nu-(m+1) / 2}}{\boldsymbol{\Gamma}_{m}(\delta / 2)} & \exp (-u \operatorname{Tr}(x)) u^{m(\delta / 2-m-1)} \\
& \times \int_{\mathcal{S}_{m}^{+}} e^{-\operatorname{Tr}\left(x^{-1} z\right) / u}{ }_{0} \mathbf{F}_{1}\left(\frac{\delta}{2} ; z\right) d z,
\end{aligned}
$$

from which we obtain the following Laplace transform

$$
\begin{aligned}
\mathbf{Q}_{x}^{(-\nu)} & {\left[\exp \left(-\lambda S_{0}\right)\right]=\lambda \int_{0}^{\infty} e^{-\lambda u} \mathbf{Q}_{x}^{(-\nu)}\left(S_{0} \leqq u\right) d u } \\
= & \frac{(\operatorname{det}(x))^{\nu-(m+1) / 2}}{\boldsymbol{\Gamma}_{m}(\delta / 2)} 2 \lambda(\lambda+\operatorname{Tr}(x))^{-\alpha / 2} \\
& \times \int_{\mathcal{S}_{m}^{+}} K_{\alpha}\left(2 \sqrt{(\lambda+\operatorname{Tr}(x)) \operatorname{Tr}\left(x^{-1} z\right)}\right)\left(\operatorname{Tr}\left(x^{-1} z\right)\right)^{\alpha / 2}{ }_{0} \mathbf{F}_{1}\left(\frac{\delta}{2} ; z\right) d z,
\end{aligned}
$$

where $\alpha=m(\delta / 2-m-1)+1, K_{\alpha}$ is the usual modified Bessel (Macdonald) function and we have used the integral representation for $K_{\alpha}$ given in formula $(5.10 .25)$ in $[19]$.

In the case where $m=1$, we obtain

$$
\mathbf{Q}_{x}^{(-\nu)}\left[\exp \left(-\lambda S_{0}\right)\right]=\frac{\lambda}{x} \frac{1}{(1+\lambda / x)^{\nu / 2}} \int_{0}^{\infty} t K_{\nu}(t \sqrt{1+\lambda / x}) I_{\nu}(t) d t
$$

by using the fact that $\widetilde{\mathbf{I}}_{\nu}(x)=I_{\nu}\left(2 z^{1 / 2}\right)$. Now, we recall the formula (cf. formula (5.15.6) in [19])

$$
\int_{0}^{\infty} t K_{\nu}(a t) I_{\nu}(t) d t=\frac{1}{a^{\nu}\left(a^{2}-1\right)}, \quad a \geqq 1
$$


from which we deduce

$$
\mathbf{Q}_{x}^{(-\nu)}\left[\exp \left(-\lambda S_{0}\right)\right]=\frac{1}{(1+\lambda / x)^{\nu}} .
$$

Hence, we again recover the well-known fact that $x S_{0}$ obeys the Gamma $(\nu)$ distribution.

Now we go back to Theorem 1.4. We may replace $t$ by any stopping time $T$ in (1.16). In particular, we may consider

$$
T_{r}=\inf \left\{t ; \operatorname{det}\left(X_{t}\right)=r\right\} \quad \text { for } \quad 0<r<\operatorname{det}(x) .
$$

We have $T_{r}<T_{0}$ a.s., and (1.16) implies

$$
\mathbf{Q}_{x}^{(-\nu)}\left[H_{T_{r}}\right]=\left(\frac{r}{\operatorname{det}(x)}\right)^{\nu} \mathbf{Q}_{x}^{(\nu)}\left[H_{T_{r}} ; T_{r}<\infty\right]
$$

for any non-negative $\left(\mathcal{F}_{t}\right)$-predictable process $\left\{H_{t}\right\}$, and, in particular, we obtain

$$
\mathbf{Q}_{x}^{(-\nu)}\left(T_{r}<\infty\right)=\left(\frac{r}{\operatorname{det}(x)}\right)^{\nu}<1 .
$$

This result is in complete agreement with the fact that $\{(\operatorname{det}(x) /$ $\left.\left.\operatorname{det}\left(X_{t}\right)\right)^{\nu}\right\}$ is a local martingale, which converges almost surely to 0 as $t \rightarrow \infty$. Therefore we obtain (see Chapter II, (3.12)Exercise, [29]), for a uniform random variable $U$,

$$
\sup _{t \geqq 0}\left(\frac{\operatorname{det}(x)}{\operatorname{det}\left(X_{t}\right)}\right)^{\nu} \stackrel{\text { (law) }}{=} \frac{1}{U} \quad \text { or } \quad \inf _{t \geqq 0} \frac{\operatorname{det}\left(X_{t}\right)}{\operatorname{det}(x)} \stackrel{\text { (law) }}{=} U^{1 / \nu} .
$$

\section{§3. Wishart Processes with Drift}

(3.1) In this section, we define Wishart processes with drift and show in particular that they are Markov processes. Recall that, in the one-dimensional case, Bessel processes with drift have been introduced by Watanabe [33] and studied by Pitman-Yor [27]. They play an essential role in the study of diffusions on $\mathbb{R}_{+}$which are invariant under time inversion. Let us first consider the case of the integral dimension, $\delta=n \in \mathbb{N}$.

Theorem 3.1. Let $\left\{B_{s}, s \geqq 0\right\}$ be an $n \times m$ Brownian matrix starting from 0 and let $\Theta=\left(\Theta_{i j}\right) \in M_{n, m}(\mathbb{R})$. Then, setting $X_{t}^{\Theta}=\left(B_{t}+\Theta t\right)^{\prime}\left(B_{t}+\Theta t\right) \equiv$ 
$\left.\widehat{\left(B_{t}+\Theta\right.} t\right)$, we have

$$
E\left[G\left(X_{t}^{\Theta}, t \leqq s\right)\right]=E\left[G\left(X_{t}, t \leqq s\right){ }_{0} \mathbf{F}_{1}\left(\frac{n}{2} ; \frac{1}{4} \hat{\Theta} X_{s}\right) \exp \left(-\frac{1}{2} \operatorname{Tr}(\hat{\Theta}) s\right)\right]
$$

for any $s>0$ and for any non-negative functional $G$, where $\hat{\Theta}=\Theta^{\prime} \Theta$ and $X_{t} \equiv X_{t}^{0}$ is an n-dimensional Wishart process.

Proof. By the usual Cameron-Martin relationship, we have

$$
\begin{aligned}
& E\left[G\left(X_{t}^{\Theta}, t \leqq s\right)\right] \\
& =E\left[G\left(X_{t}, t \leqq s\right) \exp \left(\sum_{i=1}^{n} \sum_{j=1}^{m} \Theta_{i j} B_{i j}(s)-\frac{1}{2} \sum_{i=1}^{n} \sum_{j=1}^{m}\left(\Theta_{i j}\right)^{2} s\right)\right] .
\end{aligned}
$$

Since $\sum_{i} \sum_{j} \Theta_{i j} B_{i j}(s)=\operatorname{Tr}\left(\Theta^{\prime} B_{s}\right)$, the rotational invariance of Brownian motions $(O B \stackrel{(\text { law })}{=} B$ for any $O \in O(n))$ yields

$$
\begin{aligned}
E\left[G\left(X_{t}, t \leqq s\right) \exp \left(\operatorname{Tr}\left(\Theta^{\prime} B_{s}\right)\right)\right] & \left.=E\left[G\left(\left(O B_{t}\right)^{\prime}\left(O B_{t}\right)\right), t \leqq s\right) \exp \left(\operatorname{Tr}\left(\Theta^{\prime} O B_{s}\right)\right)\right] \\
& =E\left[G\left(X_{t}, t \leqq s\right) \exp \left(\operatorname{Tr}\left(B_{s} \Theta^{\prime} O\right)\right)\right] .
\end{aligned}
$$

Since the last equality holds for any $O \in O(n)$, the integral representation (5.1) given in the appendix gives

$$
\begin{aligned}
E\left[G\left(X_{t}, t \leqq s\right) \exp \left(\operatorname{Tr}\left(\Theta^{\prime} B_{s}\right)\right)\right] & =E\left[G\left(X_{t}, t \leqq s\right) \int_{O(n)} \exp \left(\operatorname{Tr}\left(B_{s} \Theta^{\prime} O\right)\right) d O\right] \\
& =E\left[G\left(X_{t}, t \leqq s\right){ }_{0} \mathbf{F}_{1}\left(\frac{n}{2} ; \frac{1}{4} B_{s} \Theta^{\prime} \Theta B_{s}^{\prime}\right)\right]
\end{aligned}
$$

where $d O$ is the normalized Haar measure on $O(n)$. The last expression shows that the law of $\left\{X_{t}^{\Theta}\right\}$ depends on $\Theta$ only through the product $\hat{\Theta}=\Theta^{\prime} \Theta$; hence, we shall also denote $X_{t}^{\Theta}$ by $X_{t}^{(\hat{\Theta})}$. Moreover from Lemma 5.1 in the Appendix, we see

$$
E\left[G\left(X_{t}, t \leqq s\right) \exp \left(\operatorname{Tr}\left(\Theta^{\prime} B(s)\right)\right)\right]=E\left[G\left(X_{t}, t \leqq s\right)_{0} \mathbf{F}_{1}\left(\frac{n}{2} ; \frac{1}{4} \Theta X_{s} \Theta^{\prime}\right)\right] .
$$

Finally, by using Lemma 5.1 again, we obtain the better expression (3.1).

Proposition 3.2. $\quad$ (i) Keeping the notations in Theorem 3.1, the stochastic process $\left\{X_{t}^{\Theta}\right\}$ now denoted by $\left\{X_{t}^{(\hat{\Theta})}\right\}$ is a Markov process, which we 
shall refer to $\operatorname{WIS}^{(\hat{\Theta})}(n, m)$, whose transition probabilities $\mathbf{q}_{n}^{(\hat{\Theta})}(t, x, d y)$ are given by

$$
\begin{aligned}
\mathbf{q}_{n}^{(\hat{\Theta})}(t, x, d y)= & \frac{{ }_{0} \mathbf{F}_{1}(n / 2 ; \hat{\Theta} y / 4)}{{ }_{0} \mathbf{F}_{1}(n / 2 ; \hat{\Theta} x / 4)} \exp \left(-\frac{1}{2} \operatorname{Tr}(\hat{\Theta}) t\right) \mathbf{q}_{n}^{(0)}(t, x, d y) \\
= & \frac{1}{(2 t)^{n m / 2} \boldsymbol{\Gamma}_{m}(n / 2)} \exp \left(-\frac{1}{2 t} \operatorname{Tr}(x+y)\right)(\operatorname{det}(y))^{(n-m-1) / 2} \\
& \times \frac{{ }_{0} \mathbf{F}_{1}\left(n / 2 ; x y / 4 t^{2}\right){ }_{0} \mathbf{F}_{1}(n / 2 ; \hat{\Theta} y / 4)}{{ }_{0} \mathbf{F}_{1}(n / 2 ; \hat{\Theta} x / 4)} \exp \left(-\frac{1}{2} \operatorname{Tr}(\hat{\Theta}) t\right) d y .
\end{aligned}
$$

(ii) The conditional law of $B_{s}$ given $\left\{X_{t}, t \leqq s\right\}$ is given by

$$
E\left[\exp \left(\operatorname{Tr}\left(\Theta^{\prime} B_{s}\right)\right) \mid\left\{X_{t}, t \leqq s\right\}, X_{s}=y\right]={ }_{0} \mathbf{F}_{1}\left(\frac{n}{2}, \frac{\hat{\Theta} y}{4}\right) .
$$

Proof. The first assertion follows from formula (3.1), which describes $\left\{X_{t}^{(\hat{\Theta})}, t \geqq 0\right\}$ as an $h$-transform of $\left\{X_{t}, t \geqq 0\right\}$ with

$$
h\left(X_{s}, s\right)={ }_{0} \mathbf{F}_{1}\left(\frac{n}{2}, \frac{\hat{\Theta} X_{s}}{4}\right) \exp \left(-\frac{1}{2} \operatorname{Tr}(\hat{\Theta}) s\right) .
$$

In fact, we have from (3.1), for $u>s$

$$
\begin{aligned}
E\left[G\left(X_{u}^{(\hat{\Theta})}\right) \mid\right. & \left.\left\{X_{t}^{(\hat{\Theta})}, t \leqq s\right\}\right] \\
& =\frac{E\left[G\left(X_{u}\right){ }_{0} \mathbf{F}_{1}\left(n / 2 ; \hat{\Theta} X_{u} / 4\right) \exp (-\operatorname{Tr}(\hat{\Theta}) u / 2) \mid\left\{X_{t}^{(\hat{\Theta})}, t \leqq s\right\}\right]}{{ }_{0} \mathbf{F}_{1}\left(n / 2 ; \hat{\Theta} X_{s} / 4\right) \exp (-\operatorname{Tr}(\hat{\Theta}) s / 2)} \\
& =\frac{\mathbf{Q}_{u-s}^{n}\left[G(\cdot){ }_{0} \mathbf{F}_{1}(n / 2 ; \hat{\Theta} \cdot / 4)\right] \exp (-\operatorname{Tr}(\hat{\Theta})(u-s) / 2)}{{ }_{0} \mathbf{F}_{1}\left(n / 2 ; \hat{\Theta} X_{s} / 4\right)},
\end{aligned}
$$

where $\mathbf{Q}_{t}^{n}, t \geqq 0$ denotes the semigroup of the original Wishart process.

The second assertion is nothing else but (3.2).

Remark 3.3. We can also see Propositions 3.1 and 3.2 as consequences of a result by Rogers and Pitman [30]. Indeed, for $y \in \mathcal{S}_{m}^{+}$, define

$$
\Sigma(y)=\left\{\alpha \in M_{n, m}(\mathbb{R}) ; \widehat{\alpha} \equiv \alpha^{\prime} \alpha=y\right\},
$$

and let $\Lambda$ be the uniform measure on $\Sigma(y)$ given by

$$
\Lambda f(y)=\int_{O(n)} f(O \alpha) d O
$$


where $\alpha \in \Sigma(y)$ (independent of the choice of $\alpha$ ). Then, by the rotational invariance of Brownian motion, the semigroups $\mathbf{P}_{t}$ of $\left\{B_{t}\right\}$ and $\mathbf{Q}_{t}$ of $\left\{X_{t}=\widehat{B}_{t}\right\}$ satisfy

$$
\mathbf{Q}_{t} \Lambda=\Lambda \mathbf{P}_{t}
$$

Set $f_{\Theta}(\alpha)=\exp \left(\operatorname{Tr}\left(\Theta^{\prime} \alpha\right)\right)$, then the law of $B_{t}^{\Theta} \equiv B_{t}+\Theta t$, the Brownian matrix with drift $\Theta$, satisfies

$$
\mathbf{P}_{t}^{\Theta}(\alpha, d \beta)=\exp \left(-\frac{1}{2} \operatorname{Tr}(\widehat{\Theta}) t\right) \frac{f_{\Theta}(\beta)}{f_{\Theta}(\alpha)} \mathbf{P}_{t}(\alpha, d \beta) .
$$

Setting $g_{\Theta}=\Lambda f_{\Theta}$, we have (see [30])

$$
\mathbf{Q}_{t}^{\Theta}(x, d y)=\exp \left(-\frac{1}{2} \operatorname{Tr}(\widehat{\Theta}) t\right) \frac{g_{\Theta}(y)}{g_{\Theta}(x)} \mathbf{Q}_{t}(x, d y)
$$

and $\Lambda^{\Theta} \mathbf{P}_{t}^{\Theta}=\mathbf{Q}_{t}^{\Theta} \Lambda^{\Theta}$, where the kernel $\Lambda^{\Theta}$ is given by

$$
\Lambda^{\Theta}(y, d \alpha)=\frac{f_{\Theta}(\alpha)}{g_{\Theta}(y)} \Lambda(y, d \alpha) .
$$

We are now in a position to define Wishart processes with drift in general dimensions $\delta$.

Definition. Let $\delta>m-1$ and $\Delta \in \widetilde{\mathcal{S}}_{m}^{+}$. We define a Wishart process $\mathrm{WIS}^{(\Delta)}(\delta, m, x)$ of dimension $\delta$ and drift $\Delta$ as the $\widetilde{\mathcal{S}}_{m}^{+}$-valued Markov process, starting from $x$, with semigroup given by

$$
\begin{aligned}
\mathbf{q}_{\delta}^{(\Delta)}(t, x, d y)= & \frac{{ }_{0} \mathbf{F}_{1}(\delta / 2 ; \Delta y / 4)}{{ }_{0} \mathbf{F}_{1}(\delta / 2 ; \Delta x / 4)} \exp \left(-\frac{1}{2} \operatorname{Tr}(\Delta) t\right) \mathbf{q}_{\delta}^{(0)}(t, x, d y) \\
= & \frac{1}{(2 t)^{\delta m / 2} \boldsymbol{\Gamma}_{m}(\delta / 2)} \exp \left(-\frac{1}{2 t} \operatorname{Tr}(x+y)\right)(\operatorname{det}(y))^{(\delta-m-1) / 2} \\
& \quad \times \frac{{ }_{0} \mathbf{F}_{1}\left(\delta / 2 ; x y / 4 t^{2}\right){ }_{0} \mathbf{F}_{1}(\delta / 2 ; \Delta y / 4)}{{ }_{0} \mathbf{F}_{1}(\delta / 2 ; \Delta x / 4)} \exp \left(-\frac{1}{2} \operatorname{Tr}(\Delta) t\right) d y .
\end{aligned}
$$

However, we need to prove the semigroup property of $\mathbf{q}_{\delta}^{(\Delta)}$, which is done in the following.

Proposition 3.4. (i) Let $X$ be a Wishart process $\operatorname{WIS}(\delta, m, a), a \in \mathcal{S}_{m}^{+}$. Then the process $i(X)$ obtained by time inversion is a $\operatorname{WIS}^{(a)}(\delta, m, 0)$ process. (ii) More generally, if $X$ is a $\mathrm{WIS}^{(\Delta)}(\delta, m, a)$ process, then $i(X)$ is a $\mathrm{WIS}^{(a)}$ $(\delta, m, \Delta)$ process. 
Sketch of Proof. (i) After a straightforward computation, we see that the distribution of $i(X)_{t}$ is $\mathbf{q}_{\delta}^{(a)}(t, 0, d y)$ given by (3.4). Next, we compute $E\left[f\left(i(X)_{s}\right) g\left(i(X)_{t}\right)\right]$ for $s<t$ in terms of the process $X$ and the semigroup $\mathbf{q}_{\delta}(t, a, d y)$. We then obtain that $i(X)$ is a Markov process with semigroup $\mathbf{q}_{\delta}^{\prime}$ (a priori non homogeneous) given by the transition probability density

$$
\mathbf{q}_{\delta}^{\prime}(s, t ; x, y)=\frac{1}{t^{m(m+1)}} \frac{\mathbf{q}_{\delta}\left(1 / t, a, y / t^{2}\right)}{\mathbf{q}_{\delta}\left(1 / s, a, x / s^{2}\right)} \mathbf{q}_{\delta}\left(\frac{1}{s}-\frac{1}{t}, \frac{y}{t^{2}}, \frac{x}{s^{2}}\right),
$$

from which we obtain after some computations that

$$
\mathbf{q}_{\delta}^{\prime}(s, t ; x, y)=\mathbf{q}_{\delta}^{(a)}(t-s, x, y) .
$$

The proof of (ii) is similar.

Remark 3.5. The semigroup property of $\mathbf{q}^{(\Delta)}$ entails that

$$
\mathbf{L}^{\delta}\left({ }_{0} \mathbf{F}_{1}(\delta / 2 ; \Delta x / 4)\right)=\frac{1}{2} \operatorname{Tr}(\Delta){ }_{0} \mathbf{F}_{1}(\delta / 2 ; \Delta x / 4),
$$

where $\mathbf{L}^{\delta}$ denotes the infinitesimal generator of the Wishart process of dimension $\delta$. Note that the differential equations satisfied by ${ }_{0} \mathbf{F}_{1}$ given in Theorem 7.5.6, [21], in terms of eigenvalues do not directly yield (3.5). But one can translate those equations into differential equations with respect to the matrix entries.

As an application of time inversion, we give an interpretation of the Hartman-Watson distribution in terms of the Wishart processes with drift.

Proposition 3.6. Let $x, y \in \widetilde{\mathcal{S}}_{m}^{+}$and let $\mathbf{Q}_{y}^{\delta,(x)}$ denote the distribution of the Wishart process $\mathrm{WIS}^{(x)}(\delta, m, y)$ of dimension $\delta$ and drift $x$, starting from $y$. Then,

$$
\left.\mathbf{Q}_{y}^{m+1,(x)}\left[\exp \left(-\frac{\nu^{2}}{2} \int_{0}^{\infty} \operatorname{Tr}\left(X_{s}^{-1}\right) d s\right)\right)\right]=\frac{\widetilde{\mathbf{I}}_{\nu}(x y / 4)}{\widetilde{\mathbf{I}}_{0}(x y / 4)},
$$

where $\widetilde{\mathbf{I}}_{\nu}$ is defined in (1.15).

Proof. Let $f$ be a bounded function. From time inversion and the Markov property, we have

$$
\begin{aligned}
& \mathbf{Q}_{x}^{m+1}\left[f\left(X_{t}\right) \exp \left(-\frac{\nu^{2}}{2} \int_{0}^{t} \operatorname{Tr}\left(X_{u}^{-1}\right) d u\right)\right] \\
= & \mathbf{Q}_{0}^{m+1,(x)}\left[f\left(t^{2} X_{1 / t}\right) \exp \left(-\frac{\nu^{2}}{2} \int_{1 / t}^{\infty} \operatorname{Tr}\left(X_{u}^{-1}\right) d u\right)\right] \\
= & \mathbf{Q}_{0}^{m+1,(x)}\left[f\left(t^{2} X_{1 / t}\right) \mathbf{Q}_{X_{1 / t}}^{m+1,(x)}\left[\exp \left(-\frac{\nu^{2}}{2} \int_{0}^{\infty} \operatorname{Tr}\left(X_{u}^{-1}\right) d u\right)\right]\right] .
\end{aligned}
$$


On the other hand, according to (2.12), the first line of the above identities is equal to

$$
\mathbf{Q}_{x}^{m+1}\left[f\left(X_{t}\right) \frac{\widetilde{\mathbf{I}}_{\nu}\left(x X_{t} / 4 t^{2}\right)}{\widetilde{\mathbf{I}}_{0}\left(x X_{t} / 4 t^{2}\right)}\right]=\mathbf{Q}_{0}^{m+1,(x)}\left[f\left(t^{2} X_{1 / t}\right) \frac{\widetilde{\mathbf{I}}_{\nu}\left(x X_{1 / t} / 4\right)}{\widetilde{\mathbf{I}}_{0}\left(x X_{1 / t} / 4\right)}\right] .
$$

By comparison of the last two terms in (3.7) and (3.8), we obtain (3.6).

Remark 3.7. We also note that, by time inversion, the left hand side of (3.6) equals

$$
\mathbf{Q}_{x}^{m+1,(y)}\left[\exp \left(-\frac{\nu^{2}}{2} \int_{0}^{\infty} \operatorname{Tr}\left(X_{s}^{-1}\right) d s\right)\right],
$$

from which we deduce the identity

$$
\frac{\widetilde{\mathbf{I}}_{\nu}(x y)}{\widetilde{\mathbf{I}}_{0}(x y)}=\frac{\widetilde{\mathbf{I}}_{\nu}(y x)}{\widetilde{\mathbf{I}}_{0}(y x)} .
$$

But, in fact, independently from the preceding probabilistic argument, the equality $\widetilde{\mathbf{I}}_{\mu}(x y)=\widetilde{\mathbf{I}}_{\mu}(y x)$ holds as a consequence of the property that $\widetilde{\mathbf{I}}_{\mu}(z)$ depends only on the eigenvalues of the matrix $z$ (we apply this remark to both $\mu=\nu$ and $\mu=0)$.

Proposition 3.6 is a particular relation between the Wishart bridge and the Wishart process with drift. We refer to Theorem 5.8 in [27] for other relations in the Bessel case which can be extended in our context.

(3.2) Intertwining property The extension for Wishart processes of the intertwining relation (1.7) is given in the following proposition, which M.F. Bru in [6] predicted, from the results in [35], that it would hold.

To mention the result, we recall the multivariate Beta distribution. We say that $\beta_{\delta / 2, \delta^{\prime} / 2}$ is a $\operatorname{Beta}_{m}$ variable with parameter $\left(\delta / 2, \delta^{\prime} / 2\right)$ if its probability density function is given by

$$
\frac{\Gamma_{m}\left(\left(\delta+\delta^{\prime}\right) / 2\right)}{\Gamma_{m}(\delta / 2) \Gamma_{m}\left(\delta^{\prime} / 2\right)}(\operatorname{det} U)^{(\delta-m-1) / 2}\left(\operatorname{det}\left(I_{m}-U\right)\right)^{\left(\delta^{\prime}-m-1\right) / 2}, \quad 0<U<I_{m} .
$$

For details, see page 110 in [21].

Proposition 3.8. For $\delta, \delta^{\prime} \geqq m-1$ and every $t$,

$$
\mathbf{Q}_{t}^{\delta+\delta^{\prime}} \Lambda_{\delta, \delta^{\prime}}=\Lambda_{\delta, \delta^{\prime}} \mathbf{Q}_{t}^{\delta},
$$


where, letting $\beta_{\delta / 2, \delta^{\prime} / 2}$ be a Beta $_{m}$ variable with parameter $\left(\delta / 2, \delta^{\prime} / 2\right)$, $\Lambda_{\delta, \delta^{\prime}}(x, d y)$ denotes the kernel whose action on any bounded Borel function $f$ is given by

$$
\Lambda_{\delta, \delta^{\prime}} f(x)=E\left[f\left(\sqrt{x} \beta_{\frac{\delta}{2}, \frac{\delta^{\prime}}{2}} \sqrt{x}\right)\right], \quad x \in \widetilde{\mathcal{S}}_{m}^{+} .
$$

Note that (3.9) may be understood as a Markovian extension of the relation (5.3) given in the Appendix (see [35] in the Bessel case). Indeed, from (3.9), we have

$$
\mathbf{Q}_{t}^{\delta+\delta^{\prime}} \Lambda_{\delta, \delta^{\prime}} f(0)=\Lambda_{\delta, \delta^{\prime}} \mathbf{Q}_{t}^{\delta} f(0)
$$

which is equivalent to

$$
E\left[f\left(t \sqrt{\gamma_{\delta+\delta^{\prime}}} \beta_{\delta / 2, \delta^{\prime} / 2} \sqrt{\gamma_{\delta+\delta^{\prime}}}\right)\right]=E\left[f\left(t \gamma_{\delta}\right)\right]
$$

where $\gamma_{p}$ is a Wishart distribution $W_{m}\left(p, I_{m}\right), \beta$ is a Beta $m$ variable (see (5.b)) and, on the left-hand side, the two random variables are independent.

Proof. At least two proofs may be given for this result.

(i) an analytical proof, in which we just check that the Laplace transforms of both hand sides of (3.9) are equal. Indeed, take $f_{\Theta}(x)=\exp (-\operatorname{Tr}(\Theta x))$ with $\Theta \in \mathcal{S}_{m}^{+}$. We compute $\Lambda_{\delta, \delta^{\prime}} \mathbf{Q}_{t}^{\delta} f_{\Theta}(x)$ using (1.12).

On the other hand, using Theorem 7.4.2 in [21], we have

$$
\begin{aligned}
\Lambda_{\delta, \delta^{\prime}} f_{\Theta}(x) & =E\left[\exp \left(-\operatorname{Tr}\left(\Theta \sqrt{x} \beta_{\delta / 2, \delta^{\prime} / 2} \sqrt{x}\right)\right)\right. \\
& ={ }_{1} F_{1}\left(\delta / 2 ;\left(\delta+\delta^{\prime}\right) / 2 ; \sqrt{x} \Theta \sqrt{x}\right) \\
& ={ }_{1} F_{1}\left(\delta / 2 ;\left(\delta+\delta^{\prime}\right) / 2 ; \sqrt{\Theta} x \sqrt{\Theta}\right) \\
& =E\left[\exp \left(-\operatorname{Tr}\left(\sqrt{\Theta} \beta_{\delta / 2, \delta^{\prime} / 2} \sqrt{\Theta} x\right)\right)\right.
\end{aligned}
$$

We then use (1.12) again to compute $\mathbf{Q}_{t}^{\delta+\delta^{\prime}} \Lambda_{\delta, \delta^{\prime}} f_{\Theta}(x)$. The equality $\mathbf{Q}_{t}^{\delta+\delta^{\prime}} \Lambda_{\delta, \delta^{\prime}} f_{\Theta}(x)=\Lambda_{\delta, \delta^{\prime}} \mathbf{Q}_{t}^{\delta} f_{\Theta}(x)$ follows from a change of variable formula in integration.

(ii) a probabilistic proof. The proof of this result follows from the same lines as the proof of the corresponding result (1.7) for the squared Bessel processes given in [7]. The main ingredients are the time inversion invariance of Wishart processes, starting from 0 , and the relation (5.3) given in the Appendix. Indeed, let $X$ and $X^{\prime}$ be two independent Wishart processes with respective dimension $\delta$ and $\delta^{\prime}$, starting at 0 . Set $Y=X+X^{\prime}, \mathcal{X}_{t}=\sigma\left\{X_{s}, X_{s}^{\prime}, s \leqq t\right\}$ and $\mathcal{Y}_{t}=\sigma\left\{Y_{s}, s \leqq t\right\}$. Then $Y$ is a Wishart process of dimension $\delta+\delta^{\prime}$ and we 
have

$$
\begin{aligned}
E\left[F\left(Y_{u}, u \leqq t\right) f\left(X_{t}\right)\right] & =E\left[F\left(u^{2} Y_{1 / u}, u \leqq t\right) f\left(t^{2} X_{1 / t}\right)\right] \\
& =E\left[E\left[F\left(u^{2} Y_{1 / u}, u \leqq t\right) \mid Y_{1 / t}\right] f\left(t^{2} X_{1 / t}\right)\right] \\
& =E\left[E\left[F\left(u^{2} Y_{1 / u}, u \leqq t \mid Y_{1 / t}\right] \Lambda_{\delta, \delta^{\prime}} f\left(t^{2} Y_{1 / t}\right)\right]\right. \\
& =E\left[F\left(u^{2} Y_{1 / u}, u \leqq t\right) \Lambda_{\delta, \delta^{\prime}} f\left(t^{2} Y_{1 / t}\right)\right] \\
& =E\left[F\left(Y_{u}, u \leqq t\right) \Lambda_{\delta, \delta^{\prime}} f\left(Y_{t}\right)\right]
\end{aligned}
$$

where we have used the Markov property of $\left\{t^{2} Y_{1 / t}\right\}$ with respect to $\mathcal{X}_{1 / t}$ for the second equality and used (5.3) for the third one. We deduce from the above equation

$$
E\left[f\left(X_{t}\right) \mid \mathcal{Y}_{t}\right]=\Lambda_{\delta, \delta^{\prime}} f\left(Y_{t}\right)
$$

which implies the intertwining relation (3.9).

\section{$\S 4$. Some Developments Ahead}

We hope that the present paper is the first of a series of two or three papers to be devoted to the topics of Wishart processes; indeed, in the present paper, we concentrated on the extension to Wishart processes of the Hartman-Watson distribution for Bessel processes, but there are many other features of Bessel processes which may also be extended to Wishart processes. What seems to be the most accessible for now are some extensions of Spitzer type limiting results, i.e., (1.5) and (1.6); for instance, in [10], we prove that

$$
\left(\frac{2}{m \ln (t)}\right)^{2} \int_{0}^{t} \operatorname{Tr}\left(X_{u}^{-1}\right) d u \underset{t \rightarrow \infty}{\stackrel{\text { (law) }}{\longrightarrow}} T_{1}(\beta),
$$

where $X$ is our $\operatorname{WIS}(m+1, m, x)$, for $x \in \widetilde{\mathcal{S}}_{m}^{+}$, and that, if $\delta>m+1$ and $X$ is a $\operatorname{WIS}(\delta, m, x)$,

$$
\frac{1}{m(\ln (t))} \int_{0}^{t} \operatorname{Tr}\left(X_{u}^{-1}\right) d u \underset{t \rightarrow \infty}{\stackrel{(\text { a.s. })}{\longrightarrow}} \frac{1}{\delta-(m+1)} .
$$

We also hope that a number of probabilistic results concerning Bessel functions, as discussed in Pitman-Yor [27], may be extended to their matrix counterparts.

For the moment, we show, a little informally, how (4.1) may be deduced from the absolute continuity relationship (1.13) in Theorem 1.2 (in the case $m=1$, this kind of arguments has been developed in Yor [38], with further 
refinements given in Pap-Yor [25], and Bentkus-Pap-Yor [1]). Indeed, with our notation, we have:

$$
\mathbf{Q}_{x}^{(0)}\left[\exp \left(-\frac{\nu^{2}}{2} \int_{0}^{t} \operatorname{Tr}\left(X_{s}^{-1}\right) d s\right)\right]=\mathbf{Q}_{x}^{(\nu)}\left[\left(\frac{\operatorname{det}(x)}{\operatorname{det}\left(X_{t}\right)}\right)^{\nu / 2}\right] .
$$

We then replace $\nu$ by $\nu / c \ln (t)$, for some constant $c$, which we shall choose later, to see

$$
\begin{aligned}
\mathbf{Q}_{x}^{(0)}[\exp & \left.\left(-\frac{\nu^{2}}{2(c \ln (t))^{2}} \int_{0}^{t} \operatorname{Tr}\left(X_{s}^{-1}\right) d s\right)\right] \\
& =\mathbf{Q}_{x}^{(\nu /(c \ln (t)))}\left[\left(\frac{\operatorname{det}(x)}{\operatorname{det}\left(X_{t}\right)}\right)^{\nu /(2 c \ln (t))}\right] \\
& \simeq \mathbf{Q}_{x}^{(0)}\left[\exp \left(-\frac{\nu}{2 c \ln (t)} \ln \left(\operatorname{det}\left(X_{t}\right)\right)\right)\right] \\
& \simeq \mathbf{Q}_{x / t}^{(0)}\left[\exp \left(-\frac{\nu}{2 c \ln (t)} \ln \left(t^{m} \operatorname{det}\left(X_{1}\right)\right)\right)\right] \\
& \longrightarrow \exp (-\nu)
\end{aligned}
$$

as $t \rightarrow \infty$ for the choice $c=m / 2$. A similar argument easily leads to (4.2), while with the weaker convergence in probability result, instead of almost sure convergence, under $\mathbf{Q}_{x}^{\delta}$, for $\delta>m+1$.

\section{$\S 5 . \quad$ Appendix}

(5.a) We recall the definition of hypergeometric functions of matrix arguments. We refer to the book of Muirhead, Chapter $7[21]$. For $a_{i} \in \mathbb{C}, b_{j} \in \mathbb{C} \backslash$ $\left\{0, \frac{1}{2}, \frac{2}{2}, \ldots, \frac{m-1}{2}\right\}$ and $X \in \mathcal{S}_{m}(\mathbb{C})$, the hypergeometric function ${ }_{p} \mathbf{F}_{q}$ is defined by

$$
{ }_{p} \mathbf{F}_{q}\left(a_{1}, \ldots, a_{p} ; b_{1}, \ldots, b_{q} ; X\right)=\sum_{k=0}^{\infty} \sum_{\kappa} \frac{\left(a_{1}\right)_{\kappa} \cdots\left(a_{p}\right)_{\kappa}}{\left(b_{1}\right)_{\kappa} \cdots\left(b_{q}\right)_{\kappa}} \frac{C_{\kappa}(X)}{\kappa !},
$$

where $\sum_{\kappa}$ denotes the summation over all partitions $\kappa=\left(k_{1}, . ., k_{m}\right), k_{1} \geqq \cdots \geqq$ $k_{m} \geqq 0$, of $k, \kappa !=k_{1} ! \cdots k_{m} !, k=\sum_{i=1}^{m} k_{i}$,

$$
(a)_{\kappa}=\prod_{i=1}^{m}\left(a-\frac{i-1}{2}\right)_{k_{i}}, \quad(a)_{k}=a(a+1) \cdots(a+k-1), \quad(a)_{0}=1 .
$$

$C_{\kappa}(X)$ is the zonal polynomial corresponding to $\kappa$, which is originally defined for $X \in \mathcal{S}_{m}(\mathbb{R})$ and is a symmetric, homogeneous polynomial of degree $k$ in the 
eigenvalues of $X$. For $X \in \mathcal{S}_{m}(\mathbb{R})$ and $Y \in \mathcal{S}_{m}^{+}$, since the eigenvalues of $Y X$ are the same as those of $\sqrt{Y} X \sqrt{Y}$, we define $C_{\kappa}(Y X)$ by

$$
C_{\kappa}(Y X)=C_{\kappa}(\sqrt{Y} X \sqrt{Y})
$$

Hence we can also define ${ }_{p} F_{q}\left(a_{1}, \cdots ; b_{1}, \cdots ; Y X\right)$. For details, see Chapter 7 of Muirhead [21]. Moreover we find in [21] that

$$
{ }_{0} \mathbf{F}_{0}(X)=\exp (\operatorname{Tr}(X)), \quad{ }_{1} \mathbf{F}_{0}(a ; X)=\operatorname{det}\left(I_{m}-X\right)^{-a}
$$

and also that, for $X \in M_{m, n}(\mathbb{R}), m \leqq n$, and for $H=\left(H_{1}: H_{2}\right) \in O(n)$ with $H_{1} \in M_{n, m}$,

$$
\int_{O(n)} \exp \left(\operatorname{Tr}\left(X H_{1}\right)\right) d H={ }_{0} \mathbf{F}_{1}\left(\frac{n}{2} ; \frac{1}{4} X X^{\prime}\right),
$$

where $d H$ is the normalized Haar measure on $O(n)$.

We also recall the definition of the multivariate gamma function $\boldsymbol{\Gamma}_{m}(\alpha)$, $\operatorname{Re}(\alpha)>(m-1) / 2$ :

$$
\boldsymbol{\Gamma}_{m}(\alpha)=\int_{\widetilde{\mathcal{S}}_{m}^{+}} \exp (-\operatorname{Tr}(A))(\operatorname{det}(A))^{\alpha-(m+1) / 2} d A .
$$

It may be worthwhile noting that the multivariate gamma function $\boldsymbol{\Gamma}_{m}(\alpha)$ is represented as a product of the usual gamma function by

$$
\boldsymbol{\Gamma}_{m}(\alpha)=\pi^{m(m-1) / 4} \prod_{i=1}^{m} \Gamma\left(\alpha-\frac{i-1}{2}\right), \quad \operatorname{Re}(\alpha)>\frac{m-1}{2} .
$$

We now give a lemma which plays an important role in Section 3.

Lemma 5.1. Let $X$ be an $m \times m$ symmetric matrix and $\Theta$ be an $n \times m$ matrix. Then, one has

$$
{ }_{0} \mathbf{F}_{1}\left(b ; \Theta X \Theta^{\prime}\right)={ }_{0} \mathbf{F}_{1}\left(b ; \Theta^{\prime} \Theta X\right)
$$

if $b \notin \mathbb{C} \backslash\left\{0, \frac{1}{2}, \frac{2}{2}, \ldots, \frac{m \vee n-1}{2}\right\}$.

Proof. Note that the argument $\Theta X \Theta^{\prime}$ on the left-hand side of (5.2) is an $n \times n$ matrix and that $\Theta^{\prime} \Theta X$ on the right-hand side is an $m \times m$ matrix. Note also that the non-zero eigenvalues of $\Theta X \Theta^{\prime}$ and $\Theta^{\prime} \Theta X$ coincide. Then, we obtain the same type of equalities for the zonal polynomials and therefore (5.2). (5.b) The beta-gamma algebra for matrices Let $X$ and $Y$ be two independent Wishart matrices with respective distributions $W_{m}\left(\delta, I_{m}\right)$ and $W_{m}\left(\delta^{\prime}, I_{m}\right)$ 
(Muirhead's notation, [21] p.85) with $\delta+\delta^{\prime}>m-1$. Then, $S=X+Y$ is invertible and the matrix $Z$ defined by $Z=S^{-1 / 2} X S^{-1 / 2}$ is a Beta $m$ distribution with parameter $\left(\delta / 2, \delta^{\prime} / 2\right)$, see [21, Def. 3.3.2] for the definition of Beta matrices. Moreover, $Z$ and $S$ are independent, see Olkin and Rubin [23, 24], Casalis and Letac [8] for an extension to Wishart distributions on symmetric cones and [2]. We thus have the following identity in law:

$$
\left(\left(X_{\delta}+X_{\delta^{\prime}}\right)^{-1 / 2} X_{\delta}\left(X_{\delta}+X_{\delta^{\prime}}\right)^{-1 / 2}, X_{\delta}+X_{\delta^{\prime}}\right) \stackrel{(\text { law })}{=}\left(X_{\delta, \delta^{\prime}}, X_{\delta+\delta^{\prime}}\right),
$$

where, on the left-hand side, $X_{\delta}$ and $X_{\delta^{\prime}}$ are independent and Wishart distributed, and, on the right-hand side, the two variables are independent and $X_{\delta, \delta^{\prime}}$ is Beta $_{m}$ distributed.

\section{Acknowledgements}

H. Matsumoto and M. Yor are very grateful for the hospitality they received in RIMS, in April-May-June 2002, where this work was started. The four authors also dedicate this paper to Géard Letac, wishing him a happy retirement, and to Marie-France Bru who started investigating about Wishart processes.

\section{References}

[1] Bentkus, V., Pap, G. and Yor, M., Optimal bounds for Cauchy approximations for the winding distribution of planar Brownian motion, J. Theoret. Probab., 16 (2003), 345-361.

[2] Bobecka, K. and Wesolowski, J., The Lukacs-Olkin-Rubin theorem without invariance of the "quotient", Studia Math., 152 (2002), 147-160.

[3] Bru, M.-F., Processus de Wishart, C. R. Acad. Sci. Paris, Sér. I, 308 (1989), 29-32.

[4] Diffusions of perturbed principal component analysis, J. Multivariate Anal., 29 (1989), 127-136.

[5] _ Wishart processes, J. Theo. Probab., 4 (1991), 725-751.

[6] Processus de Wishart, I: Introduction. Prépublication Université Paris Nord: Série Mathématique 89-3 (1989).

[7] Carmona, P., Petit, F. and Yor, M., Beta-gamma random variables and intertwining relations between certain Markov processes, Rev. Mat. Iberoamericana, 14 (1998), 311367.

[8] Casalis, M. and Letac, G., The Lukacs-Olkin-Rubin characterization of Wishart distributions on symmetric cones, Ann. Stat., 24, (1996), 763-786.

[9] Cépa, E. and Lépingle, D., Brownian particles with electrostatic repulsion on the circle: Dyson's model for unitary random matrices revisited, ESAIM Probab. Statist., 5 (2001), 203-224.

[10] Donati-Martin, C., Doumerc, Y., Matsumoto, H. and Yor, M., Some asymptotic laws for Wishart processes, In preparation (November 2003).

[11] Geman, H. and Yor, M., Bessel processes, Asian options and perpetuities, Math Finance, 3 (1993), 349-375. 
[12] Gillet, F., Etude d'algorithmes stochastiques et arbres, Ph.D thesis at IECN, Chapter II, Décember 2003.

[13] Göing-Jaeschke, A. and Yor, M., A survey and some generalizations of Bessel processes, Bernoulli, 9 (2003), 313-350.

[14] Grabiner, D., Brownian motion in a Weyl chamber, non-colliding particles, and random matrices, Ann. Inst. H. Poincaré, 35 (1999), 177-204.

[15] Herz, C. S., Bessel functions of matrix argument, Ann. Math., 61 (1955), 474-523.

[16] Hirsch, F. and Song, S., Two-parameter Bessel processes, Stochastic Proc. Appl., 83 (1999), 187-209.

[17] Kendall, D. G., The Mardia-Dryden shape distribution for triangles: a stochastic calculus approach, J. Appl. Prob., 28 (1991), 225-230.

[18] König, W. and O'Connell, N., Eigenvalues of the Laguerre process as non-colliding squared Bessel processes, Elect. Comm. in Prob., 6 (2001), 107-114.

[19] Lebedev, N. N., Special Functions and Their Applications, Dover, New York, 1972.

[20] Lévy, P., The arithmetic character of the Wishart distribution, Proc. Cambridge Philos. Soc., 44 (1948), 295-297.

[21] Muirhead, R. J., Aspects of Multivariate Statistic Theory, Wiley, New York, 1982.

[22] O'Connell, N., Random matrices, non-colliding particle system and queues, Seminaire de probabilités XXXVI, Lect. Notes in Math. 1801, 165-182, Springer-Verlag, Berlin, 2003.

[23] Olkin, I. and Rubin, H., A characterization of the Wishart distribution, Ann. Math. Stat., 33 (1962), 1272-1280.

[24] - Multivariate Beta distributions and independence properties of the Wishart distribution, Ann. Math. Stat., 35 (1964), 261-269.

[25] Pap, G. and Yor, M., The accuracy of Cauchy approximation for the windings of planar Brownian motion, Period. Math. Hungar, 41 (2000), 213-226.

[26] Pitman, J. W. and Yor, M., Processus de Bessel, et mouvement brownien, avec $\langle\langle$ drift $\rangle\rangle$, C. R. Acad. Sc. Paris, Série A, 291 (1980), 151-153.

[27] $\longrightarrow$ Bessel processes and infinitely divisible laws, in Stochastic Integrals, ed. by D. Williams, Lect. Notes in Math., 851, 285-370, Springer, Berlin, 1981.

[28] , A decomposition of Bessel bridges, Z. W., 59 (1982), 425-457.

[29] Revuz, D. and Yor, M., Continuous martingales and Brownian motion, 3rd Ed., Springer, Berlin, 1999.

[30] Rogers, L. C. G. and Pitman, J. W., Markov functions, Ann. Probab., 9 (1981), 573-582.

[31] Shiga, T. and Watanabe, S., Bessel diffusions as a one parameter family of diffusions processes, Z. W., 31 (1975), 115-124.

[32] Spitzer, F., Some theorems concerning 2-dimensional Brownian motion, Trans. Amer. Math. Soc., 87 (1958), 187-197.

[33] Watanabe, S., On time-inversion of one-dimensional diffusion processes, Z. W., 27 (1973), 37-46.

[34] Werner, W., Girsanov's transformation for $\operatorname{SLE}(\kappa, \rho)$ processes, intersection exponents and hiding exponents, Ann. Fac. Sci. Toulouse, XIII (2004), 121-147.

[35] Yor, M., Une extension markovienne de l'algèbre des lois beta-gamma. C.R. Acad. Sci. Paris Sér. I, 308 (1989), 257-260.

[36] Loi de l'indice du lacet brownien et distribution de Hartman-Watson, Z.W., 53 (1980), 71-95.

[37] _ Some aspects of Brownian motion, Part 1: Some special Functions, Lecture in math. ETH Zürich, Birkhäuser, 1992.

[38] - Generalized meanders as limits of weighted Bessel processes, and an elementary proof of Spitzer's asymptotic result on Brownian windings. Studia Sci. Math. Hungar., 33 (1997), 339-343.

[39] _ Exponential Functionals of Brownian motion, Springer-Verlag, Berlin, 2001. 